Preprints of the

Max Planck Institute for

Research on Collective Goods

Bonn 2015/13

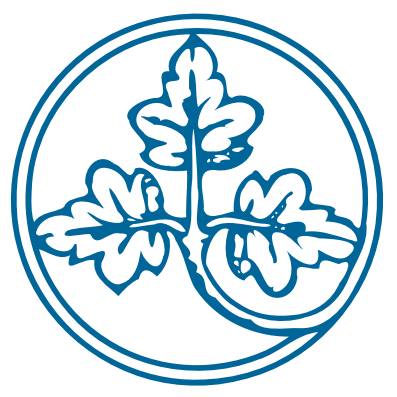

First-Order and Second-Order Ambiguity Aversion

Matthias Lang

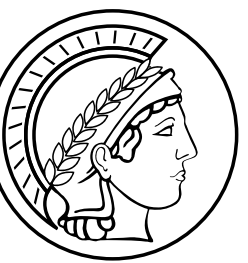




\title{
First-Order and Second-Order Ambiguity Aversion
}

\author{
Matthias Lang
}

October, 2015 


\title{
First-Order and Second-Order Ambiguity Aversion*
}

\author{
Matthias Lang**
}

October 2015

\begin{abstract}
Different models of uncertainty aversion imply strikingly different economic behavior. The key to understanding these differences lies in the dichotomy between first-order and second-order ambiguity aversion which I define here. My definition and its characterization are independent of specific representations of decisions under uncertainty. I show that with second-order ambiguity aversion a positive exposure to ambiguity is optimal if and only if there is a subjective belief such that the act's expected outcome is positive. With first-order ambiguity aversion, zero exposure to ambiguity can be optimal. Examples in finance, insurance and contracting demonstrate the economic relevance of this dichotomy.
\end{abstract}

JEL classifications: D01, D81, D82, G11

Keywords: Ambiguity, Uncertainty Aversion, Smooth Ambiguity Aversion, Subjective Beliefs, Kinked preferences

\section{Introduction}

Many decisions are made under uncertainty or ambiguity. Therefore - following the seminal contribution by Schmeidler (1989) - many models of uncertainty-averse preferences have been proposed. Recently, Cerreia-Vioglio et al. (2011) axiomatize an intriguingly general representation encompassing a wide set of uncertainty-averse preferences, in particular, Maxmin-Expected Utility (MEU), Choquet-Expected Utility with convex capacities (CEU), Smooth Ambiguity-Averse Preferences (KMM), Variational and Multiplier Preferences. Naively, one might expect uncertainty aversion to predict similar behavior in relevant economic applications independently of the specific representation. Yet, different models of uncertainty aversion arrive at strikingly different conclusions regarding economic behavior. For example, Mukerji and Tallon (2001) show with CEU preferences and idiosyncratic risk that uncertainty aversion implies no trade and incomplete markets, while Gollier (2011) derives unique equilibrium prices with Smooth Ambiguity-Averse

${ }^{*}$ I thank Sophie Bade, Han Bleichrodt, Simone Cerreia-Vioglio, Norbert Christopeit, Martin Hellwig, Christian Kellner, Asen Kochov, Eugen Kovac, Fabio Maccheroni, Massimo Marinacci, Bob Nau, Arthur Snow, and three anonymous referees for very helpful suggestions and discussions, and audiences at the ESEM 2014, RUD 2013, SFB/TR-15 2013 spring meeting, WRIEC 2010, Berlin Behavioral Economics Workshop, MPI Econ Workshop, and the Bonn University Seminar for comments.

**Free University Berlin, Department of Economics, Berlin, Germany, lang@uni-bonn.de. 

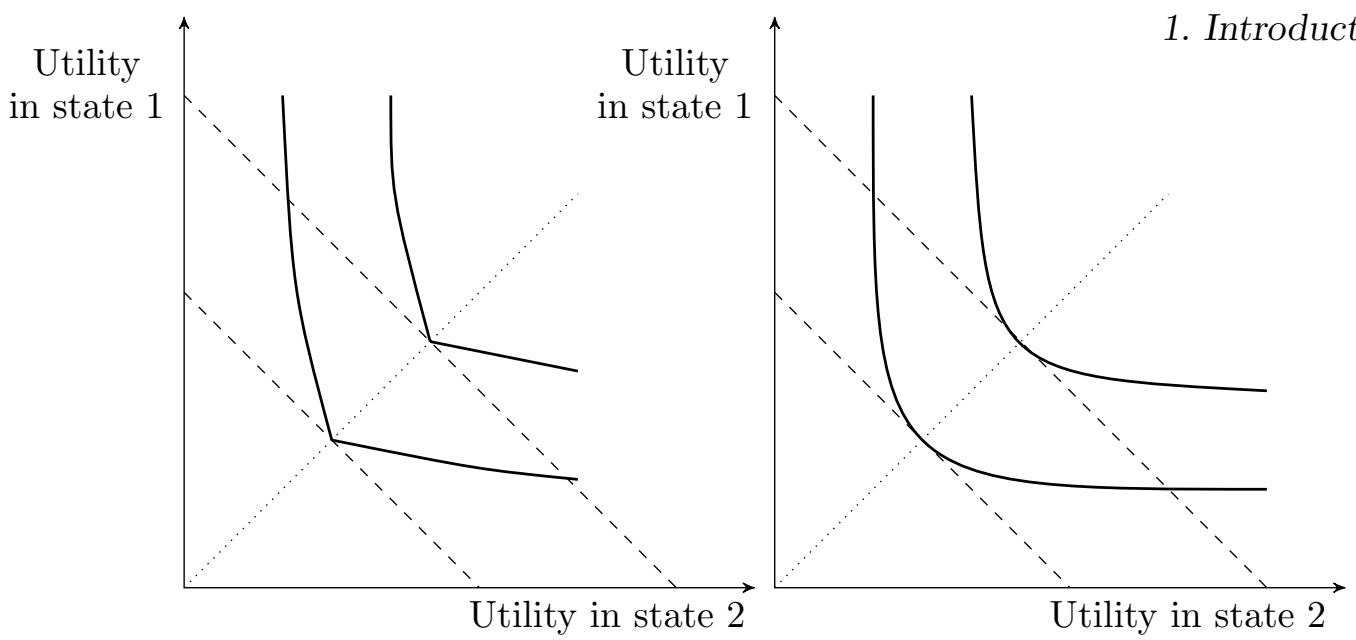

Figure 1: First-Order and Second-Order Ambiguity Aversion

Preferences. In addition, Rinaldi (2009) shows that the results of Mukerji and Tallon (2001) are not always true for Variational Preferences.

As I demonstrate in this paper, the shape of the indifference curves explains why predictions vary between different models of uncertainty aversion. In particular, the key distinction is whether indifference curves are kinked or smooth, as illustrated in Figure 1. This dichotomy relates to first-order and second-order risk aversion introduced by Segal and Spivak (1990). Second-order risk aversion implies approximately risk-neutral behavior, when small risks are concerned, while first-order risk aversion allows for riskaverse behavior, even if the stakes are small. (See Appendix A.2 for details.) I define first-order and second-order ambiguity aversion in a similar way. For this purpose, I introduce a general ambiguity premium and define first-order and second-order ambiguity aversion depending on the speed of convergence of the ambiguity premium as ambiguity disappears. This definition relates first-order and second-order ambiguity aversion to an intuitive and potentially identifiable behavioral concept, the ambiguity premium.

In addition, I characterize first-order and second-order ambiguity aversion based on the differentiability of indifference surfaces. Though intuitively appealing, this approach, however, turns out to be cumbersome, as the formal notion of differentiability of indifference surfaces is not trivial. Finally, I relate first-order and second-order ambiguity aversion to subjective beliefs a la Rigotti et al. (2008), a common concept from the literature on uncertainty-averse preferences. Unique subjective beliefs imply second-order ambiguity aversion. My definition and the characterizations are independent of any specific representation of decisions under uncertainty.

I provide a variety of examples to illustrate first-order and second-order ambiguity aversion. Some representations, such as MEU or CEU with convex capacities (pessimism), imply first-order ambiguity aversion, while others, like Smooth Ambiguity-Averse Preferences and Multiplier Preferences, imply second-order ambiguity aversion. Other representations, such as Variational or Uncertainty-Averse Preferences, exhibit both: depending on the parameters, these representations imply either first-order or second-order ambiguity aversion. 
First-order and second-order ambiguity aversion are economically important, as secondorder ambiguity aversion implies approximately uncertainty-neutral behavior for small amounts of ambiguity. This applies to, for example, investment decisions, insurance, and contracting. In contrast to second-order ambiguity aversion, first-order ambiguity aversion generally implies strikingly different predictions even if small amounts of ambiguity are involved. The dichotomy allows us to understand why the aforementioned papers arrive at different conclusions and how robust these conclusions are.

The remainder of the paper is organized as follows. Section 2 introduces the decisiontheoretic framework and a general ambiguity premium. Section 3 defines first-order and second-order ambiguity aversion and provides two alternative characterizations. Section 4 explores the consequences of the dichotomy for the optimal exposure to ambiguity and analyzes some applications of this result. Finally, Section 5 concludes. The proofs are given in Appendix A.1.

\section{Basic Definitions}

\subsection{Decision-Theoretic Framework}

Consider an individual with uncertainty-averse preferences. I operate on an Anscombe and Aumann (1963) domain with a finite state space $\Omega$, an algebra $\Sigma$ containing subsets of $\Omega$, the set of consequences $\mathbb{R}$, the set of simple lotteries over consequences $\Delta \mathbb{R}$ and the set of lotteries over states $\Delta \Omega$. An act $f$ is a measurable mapping from the state space into the simple lotteries, $f: \Omega \rightarrow \Delta \mathbb{R}$. Denote by $\mathcal{F}$ the set of all acts. Given $f \in \mathcal{F}$, $f(\omega)$ is a probability measure on $\mathbb{R}$ for any $\omega \in \Omega$, while $f(x \mid \omega)$ denotes the probability of consequence $x$ under $f(\omega)$ for any $x \in \mathbb{R}$ and $\omega \in \Omega$. Notice that any constant act is unambiguous in the sense that the act yields the same lottery $l \in \Delta \mathbb{R}$ in every state of the world. With the usual slight abuse of notation, given $l \in \Delta \mathbb{R}$, define $l \in \mathcal{F}$ to be the constant act such that $l(\omega)=l$ for all $\omega \in \Omega$. Similarly, given $x \in \mathbb{R}$, define $x \in \mathcal{F}$ to be the constant act such that $x(x \mid \omega)=1$ and $x(y \mid \omega)=0$ for all $y \neq x$ and all $\omega \in \Omega$. I model the individual's preferences on $\mathcal{F}$ by a binary relation $\succeq$. As usual, $\succ$ and $\sim$ denote respectively the asymmetric and symmetric components of $\succeq$.

Assumption 1. The individual's preferences admit an uncertainty-averse representation in the sense of Cerreia-Vioglio et al. (2011, Theorem 3) with a continuously differentiable utility index $u$.

In particular, the axioms in Cerreia-Vioglio et al. (2011) guarantee that the individual's preferences over constant acts can be represented by a von-Neumann-Morgenstern utility index $u: \mathbb{R} \rightarrow \mathbb{R}$. Differentiability of $u$ implies second-order risk attitude as stud-

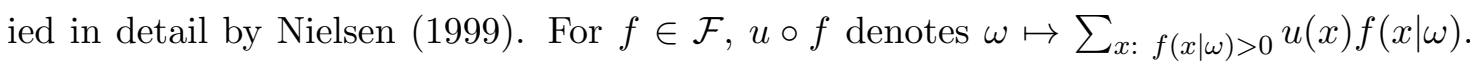
Denote the set of acts in utility space by $\overline{\mathcal{F}}=\{u \circ f \mid f \in \mathcal{F}\}$. Assumption 1 ensures that 
there is a representing utility function $U: \overline{\mathcal{F}} \rightarrow \mathbb{R}$. Denote by $i d$ the identity function with $i d(x)=x$ for all $x \in \mathbb{R}$. For $\lambda \in[0,1]$ and $f, g \in \mathcal{F}, \lambda f+(1-\lambda) g$ denotes the mixture act, i.e., $(\lambda f+(1-\lambda) g)(x \mid \omega)=\lambda f(x \mid \omega)+(1-\lambda) g(x \mid \omega)$ for all $x \in \mathbb{R}$ and all $\omega \in \Omega$. For $y \in \mathbb{R}$ and $f \in \mathcal{F}, y \oplus f$ denotes the act shifting all consequences upward by $y$, i.e., $(y \oplus f)(x \mid \omega)=f(x-y \mid \omega)$ for all $x \in \mathbb{R}$ and all $\omega \in \Omega$. Assumption 1 allows for thick indifference surfaces. Thick indifference surfaces would make the exposition considerably more complex. Therefore, I exclude thick indifference surfaces:

Assumption 2. $\epsilon \oplus f \succ f$ for all $f \in \mathcal{F}$ and all $\epsilon>0$.

Finally, for any act $f \in \mathcal{F}$, define the act $t * f$ as $(t * f)(x \mid \omega)=f(x / t \mid \omega)$ for all $x \in \mathbb{R}, \omega \in \Omega$, and $t \neq 0$. For $t=0$, define $(0 * f)$ as the degenerate distribution at 0 for all states, i.e., $(0 * f)(0 \mid \omega)=1$ for all $\omega \in \Omega$ and $(0 * f)(x \mid \omega)=0$ for all $x \neq 0$ and $\omega \in \Omega$. Hence, $t * f$ denotes the act with every consequence rescaled by $t$.

\subsection{Ambiguity Premium}

The challenge in defining the ambiguity premium lies in disentangling the risk premium and the ambiguity premium. Therefore it is impossible to follow the approach by Pratt (1964) directly. The idea is to use a benchmark lottery that captures the risk. Then the ambiguity premium of an act is the transfer that makes the individual indifferent between this benchmark lottery and the act in combination with the transfer.

For the benchmark lottery, consider wealth $w \in \mathbb{R}$, a probability measure $\nu \in \Delta \Omega$ and an act $f \in \mathcal{F}$. Now construct the constant act $l^{\nu} \in \Delta \mathbb{R}$, such that the measure $\nu$ assigns any outcome $x \in \mathbb{R}$ the same probabilities under $f$ and the lottery $l^{\nu}$

$$
l^{\nu}(x)=\sum_{\omega \in \Omega} f(x \mid \omega) \nu(\omega)
$$

for all $x \in \mathbb{R}$. Thus, a subjective expected utility maximizer with beliefs $\nu$ is indifferent between the act $f$ and the benchmark lottery $l^{\nu}$. Denote the certainty equivalent at $w$ of the lottery $l^{\nu}$ by $\mathrm{CE}_{w}\left(l^{\nu}\right)$, such that $\left(w-\mathrm{CE}_{w}\left(l^{\nu}\right)\right) \oplus l^{\nu} \sim w$. Notice that this definition of the certainty equivalent $\mathrm{CE}_{w}\left(l^{\nu}\right)$ depends on the wealth level $w$.

Definition. The ambiguity premium of an act $f \in \mathcal{F}$ at wealth $w \in \mathbb{R}$ and beliefs $\nu \in \Delta \Omega$ is a transfer $\pi_{f, w}^{\nu} \in \mathbb{R}$, such that $w \sim\left(w+\pi_{f, w}^{\nu}-\mathrm{CE}_{w}\left(l^{\nu}\right)\right) \oplus f$.

The ambiguity premium is the transfer $\pi_{f, w}^{\nu}$ that makes the individual indifferent between her endowment $w$ and the act $f$ in combination with her endowment and a transfer of $\pi_{f, w}^{\nu}-\mathrm{CE}_{w}\left(l^{\nu}\right)$. Compare this to the risk premium $\hat{\pi}_{f, w}$ of an act $f$ at wealth $w$, commonly defined as $w \sim\left(w+\hat{\pi}_{f, w}-\mathbb{E}(f)\right) \oplus f$ in a state space with objective probabilities. In the definition of the ambiguity premium, the certainty equivalent of the lottery $l^{\nu}$ replaces the expectation. The certainty equivalent ensures that the ambiguity premium does not cover the risk, but only captures the ambiguity. As $l^{\nu}$ depends on the measure $\nu$, the ambiguity premium $\pi_{f, w}^{\nu}$ also depends on $\nu$. This definition is also consistent with definitions 
for particular representations, like Maccheroni et al. (2013) for Smooth Ambiguity-Averse Preferences. To vary the amount of ambiguity, consider the ambiguity premium $\pi_{f^{t}, w}^{\nu}$ of the act $f^{t}=t f+(1-t) l^{\nu}$ for $t \in[0,1]$. The ambiguity premium vanishes as the ambiguity disappears for $t \searrow 0$.

Lemma 1. $\lim _{t \searrow 0} \pi_{f^{t}, w}^{\nu}=0$ for all beliefs $\nu \in \Delta \Omega$, acts $f \in \mathcal{F}$, and wealth levels $w \in \mathbb{R}$.

Although the ambiguity premium is defined for general beliefs, it is necessary for the results in this paper to restrict beliefs. In particular, consider the subjective beliefs by Rigotti et al. (2008) which I introduce below. The subjective beliefs are the odds at which the individual is willing to make small bets. Ramsey (1931), de Finetti (1937), and Savage (1954) consider this kind of beliefs. In the absence of ambiguity, Yaari (1969) formalizes them. In my framework, subjective beliefs by Rigotti et al. (2008) are defined as follows:

Definition. The subjective beliefs for an act $\bar{f} \in \overline{\mathcal{F}}$ are the set of (normalized) supporting hyperplanes of the upper contour set of $\bar{f}$, i.e.,

$$
\left\{P \in \Delta \Omega \mid \sum_{\omega \in \Omega} \bar{f}(\omega) P(\omega) \leq \sum_{\omega \in \Omega} \bar{g}(\omega) P(\omega) \text { for all } \bar{g} \in \overline{\mathcal{F}} \text { with } U(\bar{g}) \geq U(\bar{f})\right\} .
$$

To simplify the exposition, define subjective beliefs at a wealth level.

Definition. The subjective beliefs at wealth $w \in \mathbb{R}$ are the subjective beliefs for $u \circ w$.

The notion of subjective beliefs allows to relate uncertainty-averse preferences to ambiguity aversion in the sense of Ghirardato and Marinacci (2002). Cerreia-Vioglio et al. (2012) show that uncertainty-averse preferences are ambiguity averse in the sense of Ghirardato and Marinacci (2002) if the intersection of the subjective beliefs at all wealth levels is nonempty.

\section{$3 \quad$ First-Order and Second-Order Ambiguity Aversion}

Following the ideas in Segal and Spivak (1990) for risk, I use the ambiguity premium to define first-order and second-order ambiguity aversion. The definition depends on the speed of convergence for small amounts of ambiguity. In order to distinguish between first-order and second-order ambiguity aversion, consider the following limit

$$
L_{f, w}^{\nu}=\lim _{t \searrow 0} \frac{1}{t} \pi_{f^{t}, w}^{\nu}
$$

I will distinguish $L_{f, w}^{\nu}=0$ and $L_{f, w}^{\nu} \neq 0$. I interpret $L_{f, w}^{\nu}=0$ as 'fast' convergence of the ambiguity premium and $L_{f, w}^{\nu} \neq 0$ as 'slow' convergence of the ambiguity premium. Notice that $L_{f, w}^{\nu} \neq 0$ implies $\lim \sup _{t \searrow 0} \frac{1}{t} \pi_{f^{t}, w}^{\nu}>0$, but does not imply $L_{f, w}^{\nu}>0$. The definition of second-order risk aversion in Segal and Spivak (1990), in addition, requires 
a positive second derivative of the risk premium to distinguish even higher orders of risk aversion. It is, however, common to subsume all higher risk orders under second-order risk aversion and neglect this condition. I will do similarly and provide a definition, so that preferences are either first-order or second-order ambiguity averse. Yet, in principle, the ambiguity premium allows to define higher orders of uncertainty aversion.

Definition. There is second-order ambiguity aversion at a wealth $w \in \mathbb{R}$ if and only if $L_{f, w}^{\nu}=0$ for all subjective beliefs $\nu$ at wealth $w$ and all acts $f \in \mathcal{F}$.

Hence, there is first-order ambiguity aversion at $w$ if $L_{f, w}^{\nu} \neq 0$ for a subjective belief $\nu$ at wealth $w$ and an act $f$. To simplify the determination of first-order and secondorder ambiguity aversion and to characterize their properties, I provide two different characterizations of first-order and second-order ambiguity aversion. The first one is based on subjective beliefs as discussed at the end of Section 2.2. Here, uniqueness of the subjective beliefs matters.

Theorem 1. Suppose Assumption 1 and 2 hold. There is second-order ambiguity aversion at wealth $w \in \mathbb{R}$ if and only if there is a unique subjective belief at wealth $w$.

Hence, I refer to the subjective beliefs at a wealth $w$ from now on if there is secondorder ambiguity aversion at that wealth. Theorem 1 ensures that this notion is well defined. In addition, the theorem shows that there is first-order ambiguity aversion at a wealth $w$ if and only if there are several subjective beliefs at that wealth.

The second alternative is based on the differentiability of the indifference surface in the contingent utility space in which simple lotteries are represented by their utilities. This characterization follows the intuition in Figure 1 in the introduction. For this purpose, we need to define a notion of differentiability of an indifference surface. An indifference surface is differentiable at an act $\bar{f} \in \overline{\mathcal{F}}$ if there is a function $\eta: \mathbb{R}^{|\Omega|} \rightarrow \mathbb{R}$, so that the indifference surface is the level set of $\eta$ at the value $\eta(\bar{f})$ and $\eta$ is differentiable at $\bar{f}$ with a gradient $\nabla_{\eta}^{\bar{f}} \neq \mathbf{0}$. If an indifference surface is not differentiable at $\bar{f}$, I call it kinked at $\bar{f}$.

Theorem 2. Suppose Assumption 1 and 2 hold. There is second-order ambiguity aversion at wealth $w \in \mathbb{R}$ if and only if the indifference surface is differentiable at the act $u \circ w$.

Hence, there is first-order ambiguity aversion if the indifference surface is kinked at the constant act $u \circ w$ confirming the intuition in Figure 1. Consider a couple of specific representations. The dichotomy is defined locally at a given wealth level, but most representations exhibit either first-order ambiguity aversion or second-order ambiguity aversion. I begin with Maxmin-Expected Utility by Gilboa and Schmeidler (1989). $\Pi$ is a nonempty and closed set of probability measures $P \in \Delta \Omega$ on the state space $\Omega$. The representation is

$$
\min _{P \in \Pi} \int_{\Omega} u \circ f \mathrm{~d} P
$$

Page 6 of 27 
This representation also covers the case of Choquet-Expected Utility with a convex capacity by Schmeidler (1989).

Lemma 2. Suppose the set $\Pi$ of priors is a nonsingleton. Then MEU preferences exhibit first-order ambiguity aversion.

The intuition is the following. Whenever the ordering of the payoffs changes, this might yield different priors minimizing and maximizing expected utility. Hence, the ambiguity premium converges slowly for some acts. I restrict attention to MEU preferences and do not consider $\alpha$-MEU preferences by Ghirardato et al. (2004) here, because not all $\alpha$-MEU preferences satisfy Assumption 1. In particular, these preferences might violate convexity (Cerreia-Vioglio et al., 2011, Axiom A.3).

The next model is Smooth Ambiguity-Averse Preferences by Klibanoff et al. (2005) which is frequently used in applications (See, e.g., Kellner (2015), Maccheroni et al. (2013), or Gollier (2011)). Again $\Pi$ is a set of first-order probability measures $P \in \Delta \Omega$ on the state space $\Omega . \mu$ denotes a second-order probability measure defined on the set of priors $\Pi$. In addition, there is an increasing and concave ambiguity index $\phi$. The representation is

$$
\int_{\Pi} \phi\left(\int_{\Omega} u \circ f \mathrm{~d} P\right) \mathrm{d} \mu
$$

Lemma 3. Suppose $\phi$ is differentiable and concave. Then Smooth Ambiguity-Averse Preferences exhibit second-order ambiguity aversion.

Now consider Uncertainty-Averse Preferences by Cerreia-Vioglio et al. (2011). This is the most general representation in my framework. The Uncertainty-Averse Preference model uses a quasiconvex and linearly continuous ambiguity index $G: u(\mathbb{R}) \times \Delta \Omega \rightarrow \mathbb{R}$ that is increasing in the first argument. The representation is

$$
\inf _{P \in \Delta \Omega} G\left(\int_{\Omega} u \circ f \mathrm{~d} P, P\right)
$$

Lemma 4. Uncertainty-Averse Preferences exhibit second-order ambiguity aversion at wealth $w$ if and only if the set $\arg \inf _{P \in \Delta \Omega} G(u(w), P)$ is a singleton.

Hence, Uncertainty-Averse Preferences feature second-order ambiguity aversion at a wealth level if $G$ has a unique infimum in its second argument at this wealth. Otherwise, these preferences feature first-order ambiguity aversion at this wealth. In particular, the order of uncertainty aversion might change with the wealth. Table 1 also categorizes Variational Preferences by Maccheroni et al. (2006), Multiplier Preferences and Constraint Preferences by Hansen and Sargent (2001), Confidence Preferences by Chateauneuf and Faro (2009) and other representations of ambiguity-averse preferences. The proofs of these results are available from the author upon request. Now consider the behavioral consequences of the dichotomy between first-order and second-order ambiguity aversion. 


\begin{tabular}{c|c} 
First-Order Ambiguity Aversion & Second-Order Ambiguity Aversion \\
\hline Maxmin-Expected Utility & Multiplier Preferences \\
Choquet-Expected Utility with convex capacities & Smooth Ambiguity-Averse Preferences \\
Constraint Preferences & \\
Variational Preferences & Variational Preferences \\
Confidence Preferences & Confidence Preferences \\
Uncertainty-Averse Preferences & Uncertainty-Averse Preferences
\end{tabular}

Most representations feature either first-order or second-order ambiguity aversion. In some representations, the order of ambiguity aversion depends on parameters. Those representations are listed in both columns. The preferences in Nau (2006), Chew and Sagi (2008), Seo (2009), and Ergin and Gul (2009) are second-order ambiguity averse.

Table 1: Examples for First-Order and Second-Order Ambiguity Aversion

\section{First/Second-Order Ambiguity Aversion in Applications}

It turns out that second-order ambiguity aversion with respect to specific decisions is locally well approximated by an expected-value criterion. This local similarity is is analog to similar properties of second-order risk aversion. To formalize this idea, consider an act $f \in \mathcal{F}$. Remember that $t * f$ denotes the act with every consequence rescaled by $t$. An individual with an endowment of $w \in \mathbb{R}$ chooses the optimal $t \geq 0$ for the act

$$
w \oplus(t * f) .
$$

In addition, I assume that the individual is risk-averse.

Assumption 3. The utility index $u$ is strictly concave.

Theorem 5 in the appendix shows which results are feasible without Assumption 3. It is useful to consider also this case, because empirical research shows that not everyone has a concave utility function and prospect theory assumes S-shaped utility.

Theorem 3. Suppose Assumption 1-3 hold and $i d \circ f$ is not constantly zero. With secondorder ambiguity aversion at $w, t>0$ is optimal if and only if the subjective belief $\nu$ at wealth $w$ yields a positive expectation of the act, $\mathbb{E}_{\nu}(i d \circ f)>0$. With first-order ambiguity aversion at $w$, if $t>0$ is optimal, any subjective beliefs $\nu$ at wealth $w$ yield a positive expectation of the act, $\mathbb{E}_{\nu}(i d \circ f)>0$.

Remember that Theorem 1 shows that there is a unique subjective belief for secondorder ambiguity aversion. Therefore the condition in the first statement of Theorem 3 is well defined. First-order ambiguity aversion has additional behavioral implications.

Theorem 4. Suppose Assumptions 1-3 hold. With first-order ambiguity aversion at w, there are acts $g \in \mathcal{F}$ such that the individual facing the act $w \oplus(t * g)$ chooses $t=0$ and there is a subjective belief $\nu$ at wealth $w$ that yields a positive expectation of the act, $\mathbb{E}_{\nu}(i d \circ g)>0$. For any atomless measure with full support on $\mathcal{F}$, the set of these acts has positive mass. 
Theorem 4 implies that an individual might prefer a zero exposure to ambiguity by choosing $t=0$ even if the act's expected outcome is positive given one of the individual's subjective beliefs. Notice that uncertainty aversion changes the individual's optimal exposure $t$ to ambiguity, independently of first-order or second-order ambiguity aversion. I now turn to some applications of the dichotomy in which I suppose Assumption 1-3 hold.

\subsection{Investment}

Consider an asset $f \in \mathcal{F}$. An individual with an endowment of $w \in \mathbb{R}$ can buy or sell $t$ units of the asset $f$ at an exogenously given price per unit, $p$. Suppose that the remaining part of the individual's wealth is invested in an unambiguous asset with return $r>0$. Hence the individual chooses the optimal $t \in \mathbb{R}$ for the act

$$
r(w-p t) \oplus(t * f)
$$

Now hold the price $p$ and the individual's endowment fixed and vary the asset $f$.

Lemma 5. With second-order ambiguity aversion at rw, the individual generically buys or sells a positive amount of the asset. With first-order ambiguity aversion at rw, the individual does not buy or sell some assets. The set of these assets has positive mass in $\mathcal{F}$.

The notion 'generically' excludes a set of assets of measure zero where the individual is indifferent between trading and not trading. The proof of the lemma is based on the following corollary of Theorem 3 where the individual chooses the optimal $t \geq 0$. The result of the corollary for the special case of Smooth Ambiguity-Averse Preferences corresponds to Lemma 1 in Gollier (2011).

Corollary 1. Suppose id $\circ f$ is not constant. With second-order ambiguity aversion at $r w$, the individual invests in an asset if and only if under the subjective beliefs the expected return of the asset is higher than the unambiguous return $r$. First-order ambiguity aversion at rw can make the individual avoid any investment in the asset, even if under a subjective belief the expected return of the asset is higher than the unambiguous return $r$.

With second-order ambiguity aversion, the assets that the individual does not buy coincides with the individual's indifference surface at her endowment. These assets are negligible compared to all assets, as these assets have no mass in the asset space. Hence, uncertainty aversion in general does not yield no-trade results. See also Rigotti and Shannon (2012) and Mandler (2013) who, for example, document and discuss the effects of uncertainty aversion for asset markets for specific representations. In addition, Epstein and Schneider (2010, pp. 316,337) discuss the robustness of no-trade results depending on the representation. This corollary, in particular, implies that the no-trade results in Dow and Werlang (1992) and the incompleteness of financial markets in Mukerji and Tallon (2001) require first-order ambiguity aversion. The next section considers insurance. 


\subsection{Insurance}

Consider an individual who faces a potential loss $L \in \mathcal{F}$ that is ambiguous, i.e., $i d \circ L$ is not constant. Her endowment is $w \in \mathbb{R}$. She can buy an insurance contract covering a fraction $\alpha \geq 0$ of the loss. The insurer requests a constant premium $p$ per unit of coverage $\alpha$. Therefore buying an insurance contract with a coverage $\alpha$ corresponds to the act

$$
(w-\alpha p) \oplus((1-\alpha) * L) .
$$

Lemma 6. With second-order ambiguity aversion at $w-p$, full insurance coverage is demanded at a unique premium. With first-order ambiguity aversion at $w-p$, demand for full insurance coverage is consistent with an interval of premium levels.

Again the proof uses a corollary of Theorem 3 .

Corollary 2. Consider a subjective belief $\nu$ at wealth $w-p$. Suppose $\mathbb{E}_{\nu}(i d \circ L)<0$. With second-order ambiguity aversion at $w-p$, (at least) full insurance coverage is demanded if and only if the premium $p$ is lower than $\left|\mathbb{E}_{\nu}(i d \circ L)\right|$. Exactly full insurance coverage is demanded at the premium $p=\left|\mathbb{E}_{\nu}(i d \circ L)\right|$. With first-order ambiguity aversion at $w-p$, demand for full insurance coverage is consistent with premium levels $p$ above $\left|\mathbb{E}_{\nu}(i d \circ L)\right|$.

Notice that, according to the corollary, Mossin's (1968) Theorem on the optimality of full coverage versus partial coverage still holds with second-order ambiguity aversion. Similar results are valid if the individual can self-insure or invest into prevention effort. I assume that prevention effort is costly with strictly increasing, convex and differentiable $\operatorname{costs} c(\cdot)$. Thus, the individual chooses $e \in[0,1]$ for the act

$$
(w-c(e)) \oplus((1-e) * L) .
$$

Corollary 3. Consider a subjective belief $\nu$ at wealth $w-c(1)$. Suppose $\mathbb{E}_{\nu}(i d \circ L)<0$. With second-order ambiguity aversion at wealth $w-c(1)$, the risk of a potential loss will be completely eliminated if and only if the marginal costs of the last prevention effort are lower than $\left|\mathbb{E}_{\nu}(L)\right|$. With first-order ambiguity aversion at wealth $w-c(1)$, the individual might be willing to avoid the loss completely, even if the marginal costs are higher than $\left|\mathbb{E}_{\nu}(L)\right|$.

\subsection{Contracting}

The informativeness principle of Holmström (1979) states that more informative signals help the principal. In particular, the principal's costs of implementing a given level of effort by the agent decrease with more informative signals. Holmström (1979) proves the informativeness principle for subjective expected utility maximizers. Ghirardato (1994) was the first to point out that more informative signals might hurt the principal if the agent is uncertainty averse with CEU preferences. This result violates the informativeness principle. Defining rigorously an informative signal in my general setting is beyond the scope 
of this paper. Yet, I show that some contracts that make second-order ambiguity-averse agents implement positive effort fail to incentivize first-order ambiguity-averse agents. Consider an uncertainty-averse agent working for a risk-neutral and uncertainty-neutral principal. They can enter a contract that specifies the agent's wage $W(y)$ depending on the performance $y \in Y \subset[0,1]$ with a finite set $Y$. After signing such a contract, the agent chooses effort level $e \geq 0$, which is unobservable by the principal. The effect of the agent's effort $e$ on performance $y$ is summarized in acts $y^{e} \in \mathcal{F}$ for $e \geq 0$. By assumption, any realization of $y^{e}$ has to be an element of $Y$. With a slight abuse of notation, I write $w\left(y^{e}\right)$ for the act that pays a wage $W\left(y^{e}\right)$ for each realization of $y^{e}$. The agent's payoffs are $w\left(y^{e}\right)-c(e)$. The disutility of performing effort $c(\cdot)$ is increasing and strictly convex with $c(0)=0$ and $c^{\prime}(0)=0$. In addition, $c$ is twice differentiable. The agent's preferences satisfy Assumption 1-3. In addition, assume that $y^{0}$ is constant and that for every $\epsilon>0$ there is a $\delta>0$ such that $\left\|u \circ\left(-c(e) \oplus w\left(y^{e}\right)\right)-u \circ w\left(y^{0}\right)\right\|<\epsilon$ for all $e \in[0, \delta]$ with $\|\cdot\|$ the Euclidean norm. The principal's objective function is $\mathbb{E}_{\mu^{e}}(y-W(y))$ given her prior $\mu^{e}$ on $Y$. Finally, I assume that the principal's prior $\mu^{e}$ is consistent with the agent's subjective beliefs at all wealth levels. Hence the agent chooses her optimal $e \geq 0$ for the act

$$
-c(e) \oplus w\left(y^{e}\right) .
$$

Corollary 4. Suppose that there is a $\delta>0$ such that a subjective expected utility maximizer with risk index $u$ and beliefs $\mu^{e}$ prefers $-c(e) \oplus w\left(y^{e}\right)$ to $w\left(y^{0}\right)$ for all $e \in[0, \delta]$. Then the agent exerts positive effort if she is second-order ambiguity averse at wealth $\mathbb{E}_{\mu^{0}} W(y)$. With first-order ambiguity aversion at wealth $\mathbb{E}_{\mu^{0}} W(y)$, it may be optimal for the agent not to exert any effort.

This corollary conveys the intuition that providing incentives for sufficiently small effort levels is more expensive with first-order ambiguity aversion than with second-order ambiguity aversion. To determine the optimal wage schedule, further assumptions on the preferences are necessary. Consider the following example with MEU preferences. Let $Y=\{0,1\}$ and $c(e)=e^{2} / 2$. The agent receives a reservation wage of $\bar{w}$ if she rejects the principal's offer. Instead of specifying the acts $y^{e}$, I use a reduced form here and directly consider beliefs about $y$. Assume that the set of the agent's probabilities of $y=1$ is $\Pi(e)=\left[\frac{1}{2} \mu(e), \frac{1}{2} \mu(e)+\frac{1}{2}\right]$ with the principal's prior $\mu(e)=1-\tilde{p} \exp (-e)$ and $\tilde{p} \in(0,1)$. The optimal wage scheme to implement effort $e$ is $w_{0}=c(e)+\bar{w}-\mu(e) \frac{e}{\mu^{\prime}(e)}$ for low performances, $y=0$, and $w_{1}=w_{0}+\frac{2 e}{\mu^{\prime}(e)}$ for high performances, $y=1$. The principal's expected profits are $\mu(e)\left(1-\frac{e}{\mu^{\prime}(e)}\right)-c(e)-\bar{w}$ and are decreasing in $e$ if $\tilde{p}<\frac{\sqrt{5}-1}{2}$. Then a constant wage is optimal and the principal does not give the agent any incentives. Hence, optimality of a constant wage is consistent with first-order ambiguity aversion. In contrast, a subjective expected utility maximizer, who is by definition second-order ambiguity averse, would be offered wages of $w_{0}^{N}=w_{0}$ and $w_{1}^{N}=w_{0}+\frac{e}{\mu^{\prime}(e)}$. In this case, the principal's expected profits are increasing in $e$ for sufficiently small $e$. 
Mukerji and Tallon (2004) analyze a similar cause for incomplete contracts under uncertainty aversion. They consider wage indexation with aggregate and idiosyncratic price shocks. Aggregate price shocks affect the prices of all goods in the economy, while idiosyncratic price shocks just affect a good not consumed or produced by the firm and the agents. Agents have CEU preferences with convex capacities. If idiosyncratic price shocks are sufficiently ambiguous and aggregate price shocks are sufficiently small, wages are contracted in absolute terms and no wage indexation takes places. This result requires first-order ambiguity aversion according to the previous considerations. With secondorder ambiguity aversion it is optimal to have some wage indexation. The reason is that wage indexation allows hedging some ambiguity about aggregate price shocks and the ambiguity premium for doing so is negligible.

There is another line of reasoning in the literature why uncertainty aversion makes incomplete contracts optimal. Mukerji (1998) considers a bilateral hold-up setting with CEU preferences and convex capacities. Although the first-best is implementable in a complete contract for risk-neutral agents, it is unattainable for uncertainty-averse agents. This result is robust with respect to the representation of uncertainty-averse preferences. A formal proof with second-order ambiguity aversion would proceed along the lines of Section 5 in Williams and Radner (1988), who show that for subjective expected utility maximizers the first-best is unattainable with second-order risk aversion.

\section{Conclusion}

This paper defines first-order and second-order ambiguity aversion and shows implications for decisions. In addition, it provides two alternative characterizations. The definition and its characterization are independent of specific representations of decisions under uncertainty. They unify a variety of ideas expressed in the literature, and show how the intuitive notion of kinked indifference curves can be expressed in subjective beliefs - a common notion in the literature on uncertainty aversion - and in a potentially behaviorally identifiable item such as my ambiguity premium. First-order and second-order ambiguity aversion and the ambiguity premium are novel concepts. In particular, there is no alternative notion of a general ambiguity premium available in the literature. In addition, the definition of first- and second-order ambiguity aversion refines previous crude distinctions such as those between different representations, in particular Smooth Ambiguity-Averse Preferences and MEU. Table 1 on page 8 shows how the dichotomy applies to common representations of decisions under uncertainty.

Notice that there are other relevant distinctions between ambiguity models which are independent of the dichotomy between first-order and second-order ambiguity aversion. As an example, consider the second thought experiment of Epstein (2010). A decision maker has to choose one of two acts. Ambiguity is independent, but symmetric. As 
an example think of a draw from one of two Ellsberg urns. Due to the symmetry, the decision maker is indifferent between both acts. Now consider a mixture of the two acts in the sense that the toss of a fair coin determines which of the two acts is chosen. In the MEU representation, the decision maker is indifferent between these acts and the mixture of them. In Smooth Ambiguity Aversion, the decision maker prefers the mixture of the acts. Now, turn to Variational Preferences. It is straightforward to construct Variational Preferences that exhibit first-order ambiguity aversion and at the same time prefer the mixture of the two acts to the acts themselves. Therefore First-order and second-order ambiguity aversion introduce a new dichotomy to the literature which is relevant for many economic applications. In addition, there is empirical support for the implications of this dichotomy. Ahn et al. (2014) use bunching patterns in portfolio choices to discriminate between kinked and smooth ambiguity preferences in an experiment. Their data seems to support both kind of preferences for different individuals. As discussed before, the distinction between kinked and smooth ambiguity preferences is an imperfect proxy for the distinction between first-order and second-order ambiguity aversion. This emphasizes the importance of my definition of first-order and second-order ambiguity aversion and shows that the dichotomy is open to empirical scrutiny.

I show that second-order ambiguity aversion does not change the decision whether to accept some exposure to ambiguity compared to a subjective expected utility maximizer. It does affect how much exposure the individual is willing to accept, though. This general result is applied to an investment and an insurance setting. Moreover, I study the implications of this result for contracting problems.

Finally, the definition here applies only to constant acts. It seems interesting to extend first-order and second-order ambiguity aversion to non-constant acts. In the MEU representation, for example, there could be kinks at any act that yields the same utility in at least two states of the world. Theorem 1 allows for a straightforward generalization of first-order and second-order ambiguity aversion to any act in my framework. Notice, however, that in the proofs I use the fact that the definition is restricted to constant acts. Therefore it is unclear whether all implications are still valid for such a general definition.

\section{A Appendix}

\section{A.1 Proofs}

Proof of Lemma 1: By definition of the certainty equivalent $\mathrm{CE}_{w}\left(l^{\nu}\right)$, the individual is indifferent between the constant acts $w$ and $\left(w-\mathrm{CE}_{w}\left(l^{\nu}\right)\right) \oplus l^{\nu}$. Therefore $\pi_{f^{0}, w}^{\nu}=0$. Notice that $\pi_{f^{t}, w}^{\nu}$ is defined by $U\left(u \circ\left(\left(w-\mathrm{CE}_{w}\left(l^{\nu}\right)+\pi_{f^{t}, w}^{\nu}\right) \oplus t f+(1-t) l^{\nu}\right)\right)=U(u \circ w)$. Brouwer's fixed-point theorem ensures existence of $\pi_{f^{t}, w}^{\nu}$, because $U$ is continuous under Assumption 1 (Cerreia-Vioglio et al., 2011, Lemma 57). Assumption 2 together with an implicit function theorem, like Kudryavtsev (1990), guarantees continuity of $\pi_{f^{t}, w}^{\nu}$ in $t$. By continuity, the limit of the ambiguity premium equals zero. 
Proof of Theorem 1: Consider a wealth $w$ and an act $f \in \mathcal{F}$. Define $\bar{w}=u(w)$. The upper contour set of $U$ at a $\bar{f}_{0} \in \overline{\mathcal{F}}$ is denoted $B\left(\bar{f}_{0}\right)=\left\{\bar{f} \in \overline{\mathcal{F}} \mid U(\bar{f}) \geq U\left(\bar{f}_{0}\right)\right\}$. Without loss of generality suppose $0 \notin B(\bar{w})$. Now define the function $\tilde{W}: \overline{\mathcal{F}} \rightarrow \overline{\mathbb{R}}$ with

$$
\tilde{W}(\bar{f})= \begin{cases}-\inf \left\{\alpha \in \mathbb{R}_{+} \mid \alpha \bar{f} \in B(\bar{w})\right\} & \text { if } \exists \alpha \in \mathbb{R}_{+}: \alpha \bar{f} \in B(\bar{w}) \\ -\infty & \text { otherwise. }\end{cases}
$$

Notice that $\overline{\mathcal{F}} \subseteq \mathbb{R}^{\Omega}$ is homeomorphic to a corresponding subspace of $\mathbb{R}^{|\Omega|}$. Hence, addition, scalar-multiplication, and the inner product in $\overline{\mathcal{F}}$ are well-defined.

To verify that $\tilde{W}$ is concave, consider $\bar{f}_{1}, \bar{f}_{2} \in \overline{\mathcal{F}}$ with $\tilde{W}\left(\bar{f}_{1}\right), \tilde{W}\left(\bar{f}_{2}\right)>-\infty$ first. Cerreia-Vioglio et al. (2011, Lemma 57) show that the representing utility function $U$ is monotone, quasiconcave and continuous under Assumption 1. A quasiconcave and continuous function has closed and convex upper contour sets. Therefore $B(\bar{w})$ is a closed and convex set. As $\overline{\mathcal{F}}$ is a Hilbert space, the closest point theorem ensures $\left(-\tilde{W}\left(\bar{f}_{i}\right)\right) \bar{f}_{i} \in$ $B(\bar{w})$ for $i=1,2$. Convexity of $B(\bar{w})$ implies $-\lambda \tilde{W}\left(\bar{f}_{1}\right) \bar{f}_{1}-(1-\lambda) \tilde{W}\left(\bar{f}_{2}\right) \bar{f}_{2} \in B(\bar{w})$ for all $\lambda \in[0,1]$. Moreover,

$$
-\left(\lambda \tilde{W}\left(\bar{f}_{1}\right)+(1-\lambda) \tilde{W}\left(\bar{f}_{2}\right)\right)\left(\lambda^{\prime} \bar{f}_{1}+\left(1-\lambda^{\prime}\right) \bar{f}_{2}\right)=-\lambda \tilde{W}\left(\bar{f}_{1}\right) \bar{f}_{1}-(1-\lambda) \tilde{W}\left(\bar{f}_{2}\right) \bar{f}_{2}
$$

with $\lambda=\frac{\lambda^{\prime} \tilde{W}\left(\bar{f}_{2}\right)}{\lambda^{\prime} \tilde{W}\left(\bar{f}_{2}\right)+\left(1-\lambda^{\prime}\right) \tilde{W}\left(\bar{f}_{1}\right)} \in[0,1]$ for all $\lambda^{\prime} \in[0,1]$. Hence, there is a $\tilde{\alpha}=$ $\lambda \tilde{W}\left(\bar{f}_{1}\right)+(1-\lambda) \tilde{W}\left(\bar{f}_{2}\right)$ such that

$$
-\tilde{\alpha}\left(\lambda^{\prime} \bar{f}_{1}+\left(1-\lambda^{\prime}\right) \bar{f}_{2}\right) \in B(\bar{w}) .
$$

Notice that

$$
\begin{aligned}
\lambda \geq \lambda^{\prime} & \Leftrightarrow \frac{\lambda^{\prime} \tilde{W}\left(\bar{f}_{2}\right)}{\lambda^{\prime} \tilde{W}\left(\bar{f}_{2}\right)+\left(1-\lambda^{\prime}\right) \tilde{W}\left(\bar{f}_{1}\right)} \geq \lambda^{\prime} \\
& \Leftrightarrow W\left(\bar{f}_{2}\right) \leq \lambda^{\prime} \tilde{W}\left(\bar{f}_{2}\right)+\left(1-\lambda^{\prime}\right) \tilde{W}\left(\bar{f}_{1}\right) \Leftrightarrow \tilde{W}\left(\bar{f}_{2}\right) \leq \tilde{W}\left(\bar{f}_{1}\right) .
\end{aligned}
$$

Therefore,

$$
\lambda^{\prime} \tilde{W}\left(\bar{f}_{1}\right)+\left(1-\lambda^{\prime}\right) \tilde{W}\left(\bar{f}_{2}\right) \leq \lambda \tilde{W}\left(\bar{f}_{1}\right)+(1-\lambda) \tilde{W}\left(\bar{f}_{2}\right)=\tilde{\alpha} \leq \tilde{W}\left(\lambda^{\prime} \bar{f}_{1}+\left(1-\lambda^{\prime}\right) \bar{f}_{2}\right) .
$$

The last inequality follows from $(4) \cdot \lambda^{\prime} \tilde{W}\left(\bar{f}_{1}\right)+\left(1-\lambda^{\prime}\right) \tilde{W}\left(\bar{f}_{2}\right) \leq \tilde{W}\left(\lambda^{\prime} \bar{f}_{1}+\left(1-\lambda^{\prime}\right) \bar{f}_{2}\right)$ is also valid if $\tilde{W}\left(\bar{f}_{i}\right)=-\infty$ for $i=1$ or 2 . Consequently, $\tilde{W}$ is a proper concave function.

According to Rockafellar (1970, p. 308) the superdifferential at an act $\bar{f}_{0} \in \overline{\mathcal{F}}$ is the convex set of all supergradients $\bar{g}$ at $\bar{f}_{0}$ and a supergradient $\bar{g}$ of a concave function $W$ : $\overline{\mathcal{F}} \rightarrow \mathbb{R}$ at $\bar{f}_{0}$ is any $\bar{g} \in \overline{\mathcal{F}}$ with the property $W(\bar{f})-W\left(\bar{f}_{0}\right) \leq\left\langle\bar{g}, \bar{f}-\bar{f}_{0}\right\rangle$ for all $\bar{f} \in \overline{\mathcal{F}}$. Here $\langle\bar{g}, \bar{f}\rangle=\sum_{\omega \in \Omega} \bar{g}(\omega) \bar{f}(\omega)$ denotes the inner product. Rockafellar (1970, Theorem 23.4) ensures that the superdifferential of $\tilde{W}$ at $\bar{w}$ exists.

Assume to the contrary that there is a supergradient $\bar{g} \in \overline{\mathcal{F}}$ of $\tilde{W}$ at $\bar{w}$ with $\bar{g}\left(\omega^{\prime}\right)<0$ for a $\omega^{\prime} \in \Omega$. Consider the act $\bar{f}^{x} \in \overline{\mathcal{F}}$ with $\bar{f}^{x}\left(\omega^{\prime}\right)=x \in \mathbb{R}$ and $\bar{f}^{x}(\omega)=\bar{w}$ for all $\omega \neq \omega^{\prime}$. By the definition of the supergradient $\tilde{W}\left(\bar{f}^{x}\right)-\tilde{W}(\bar{w}) \leq\left\langle\bar{g}, \bar{f}^{x}-\bar{w}\right\rangle=g\left(\omega^{\prime}\right)(x-\bar{w})$ for 
all $x \in \mathbb{R}$. Hence, $\tilde{W}\left(\bar{f}^{x}\right)<\tilde{W}(\bar{w})=-1$ for all $x>\bar{w}$. Yet, $\tilde{W}\left(\bar{f}^{x}\right)<-1$ for all $x>\bar{w}$ implies $\bar{f}^{x} \notin B(\bar{w})$ for all $x>\bar{w}$ contradicting monotonicity of $U$ according to CerreiaVioglio et al. (2011, Lemma 57). Therefore $\bar{g}(\omega) \geq 0$ for all $\omega \in \Omega$ for all supergradients $\bar{g} \in \overline{\mathcal{F}}$ of $\tilde{W}$ at $\bar{w}$.

Suppose the subjective beliefs at wealth $w$ are a singleton. Denote these subjective beliefs by $\nu$. Assume to the contrary that there are two different supergradients $\bar{g}, \bar{g}^{\prime} \in \overline{\mathcal{F}}$ of $\tilde{W}$ at $\bar{w}$ with $\bar{g} \neq \bar{g}^{\prime}$. The two-sided directional derivative of $\tilde{W}$ at $\bar{w}$ with respect to $\mathbf{1}=(1,1, \ldots, 1)$ is well-defined and equals $\lim _{\epsilon \rightarrow 0} \frac{1}{\epsilon}(\tilde{W}(\bar{w}+\epsilon)-\tilde{W}(\bar{w}))=\lim _{\epsilon \rightarrow 0} \frac{1}{\epsilon}\left(1-\frac{\bar{w}}{\bar{w}+\epsilon}\right)=$ $1 / \bar{w}$. Then according to Rockafellar (1970, Theorem 23.2) $\sum_{\omega \in \Omega} \bar{g}(\omega) \leq \frac{1}{\bar{w}} \leq \sum_{\omega \in \Omega} \bar{g}(\omega)$ and, hence, $\sum_{\omega \in \Omega} \bar{g}(\omega)=\frac{1}{\bar{w}}$ for all supergradients $\bar{g}$ of $\tilde{W}$ at $\bar{w}$. Therefore $\bar{g}$ and $\bar{g}^{\prime}$ cannot be parallel and $\bar{g}, \bar{g}^{\prime} \neq \mathbf{0}$. Normalize both supergradients such that $\sum_{\omega \in \Omega} \bar{g}(\omega)=$ $\sum_{\omega \in \Omega} \bar{g}^{\prime}(\omega)=1$. As $\bar{g}, \bar{g}^{\prime} \neq \mathbf{0}$ and $\bar{g}(\omega) \geq 0$ and $\bar{g}^{\prime}(\omega) \geq 0$ for all $\omega \in \Omega$ as proven in the last paragraph, this normalization is feasible. In addition, the normalized supergradients are probability measures. If after normalization $\bar{g}=\bar{g}^{\prime}$, this implies that initially $\bar{g}$ and $\bar{g}^{\prime}$ were parallel - contradicting our assumption. Therefore also after normalization $\bar{g} \neq \bar{g}^{\prime}$. Following the definition of the supergradients, $\tilde{W}(\bar{f})-\tilde{W}(\bar{w}) \leq\langle\bar{g}, \bar{f}-\bar{w}\rangle$ and $\tilde{W}(\bar{f})-\tilde{W}(\bar{w}) \leq\left\langle\bar{g}^{\prime}, \bar{f}-\bar{w}\right\rangle$ for all $\bar{f} \in B(\bar{w})$. Yet, $\bar{f} \in B(\bar{w})$ implies $\tilde{W}(\bar{f}) \geq-1$ and $\tilde{W}(\bar{f})-\tilde{W}(\bar{w}) \geq 0$. Therefore $0 \leq\langle\bar{g}, \bar{f}-\bar{w}\rangle$ and $0 \leq\left\langle\bar{g}^{\prime}, \bar{f}-\bar{w}\right\rangle$ for all $\bar{f} \in B(\bar{w})$. Consequently, $\bar{g}$ and $\bar{g}^{\prime}$ are subjective beliefs at wealth $w$ contradicting my assumption that the subjective beliefs at wealth $w$ are a singleton. This contradiction proves that the superdifferential of $\tilde{W}$ at $\bar{w}$ is a singleton.

Rockafellar (1970, Theorem 25.1) shows that a concave function has a unique supergradient at $\bar{w}$ if and only if the function is differentiable at $\bar{w}$. Therefore the function $\tilde{W}$ is differentiable at $\bar{w}$. The gradient equals $\frac{1}{\bar{w}} \nu \neq \mathbf{0}$ as we have shown above.

Denote by $f_{1}^{t} \in \mathcal{F}$ the act $\left(w-\mathrm{CE}_{w}\left(l^{\nu}\right)\right) \oplus t f+(1-t) l^{\nu}$ and by $\bar{f}_{1}^{t}=u \circ f_{1}^{t}$ its projection into $\overline{\mathcal{F}}$. Notice that

$$
\begin{aligned}
\bar{f}_{1}^{t}: \omega \mapsto \sum_{\left\{x \in \mathbb{R} \mid \min \left\{f(x \mid \omega), l^{\nu}(x)\right\}>0\right\}} u\left(w-\mathrm{CE}_{w}\left(l^{\nu}\right)+x\right)\left(t f(x \mid \omega)+(1-t) l^{\nu}(x)\right)= \\
\quad=t \sum_{\{x \in \mathbb{R} \mid f(x \mid \omega)>0\}} u\left(w-\mathrm{CE}_{w}\left(l^{\nu}\right)+x\right) f(x \mid \omega)+\underset{\left\{x \in \mathbb{R} \mid l^{\nu}(x)>0\right\}}{(1-t) \sum_{w} u\left(w-\mathrm{CE}_{w}\left(l^{\nu}\right)+x\right) l^{\nu}(x) .}
\end{aligned}
$$

Hence, $\bar{f}_{1}^{t}=t \bar{f}_{1}^{t=1}+(1-t) \bar{f}_{1}^{t=0}=t \bar{f}_{1}^{t=1}+(1-t) \bar{w}$.

Consider the distance $d_{1}(t)$ between $\bar{f}_{1}^{t}$ and the upper contour set $B(\bar{w})$ in direction $\bar{f}_{1}^{t}$

$$
\begin{aligned}
d_{1}(t) & =\inf \left\{\beta \in \mathbb{R}_{+} \mid \beta \bar{f}_{1}^{t}+\bar{f}_{1}^{t} \in B(\bar{w})\right\}\left\|\bar{f}_{1}^{t}\right\|= \\
& =\left(\inf \left\{\alpha \in \mathbb{R}_{+} \mid \alpha \bar{f}_{1}^{t} \in B(\bar{w})\right\}-1\right)\left\|\bar{f}_{1}^{t}\right\|=\left(\tilde{W}(\bar{w})-\tilde{W}\left(\bar{f}_{1}^{t}\right)\right)\left\|\bar{f}_{1}^{t}\right\|
\end{aligned}
$$

for $\tilde{W}(\cdot)$ as defined in (3) and $\|\cdot\|$ the Euclidean norm. Figure 2 depicts this distance. Suppose $\bar{f}_{1}^{t}=\bar{w}$, then $\bar{f}_{1}^{t=1}=\bar{w}$ and, hence, $\bar{f}_{1}^{t}=\bar{w}$ for all $t \in[0,1]$. In addition, $d_{1}(t)=0$ for all $t \in[0,1]$. Therefore I can focus on $\bar{f}_{1}^{t} \neq \bar{w}$. The first part of the proof shows that 


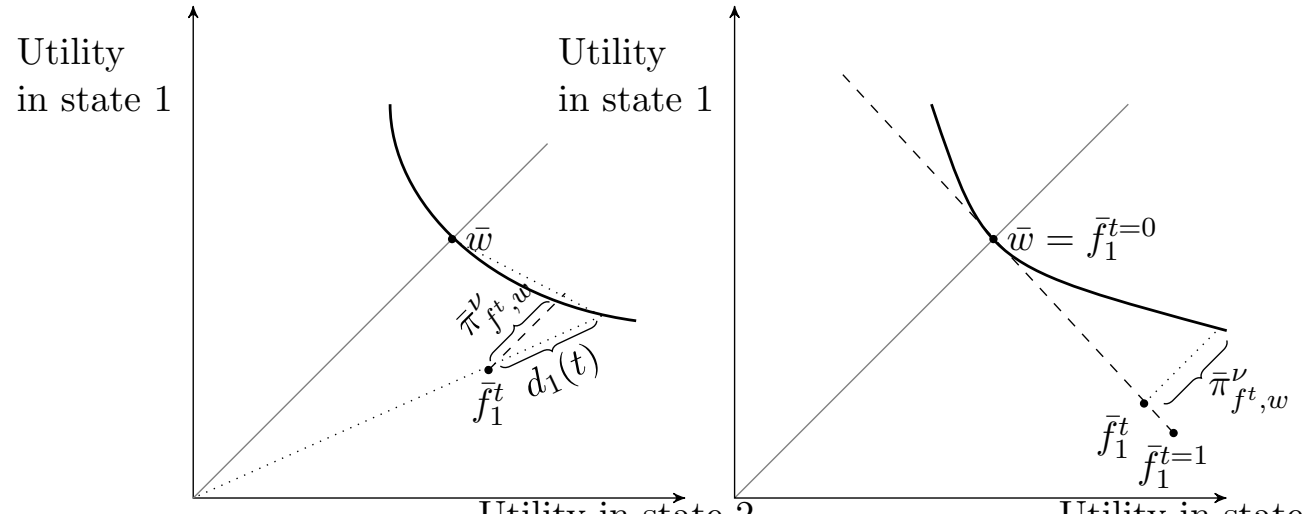

Utility in state 2

Utility in state 2

The figure depicts a contingent utility space for two states of the world. $\bar{w}$ denotes the constant act with utility $u(w)$, while $\bar{f}_{1}^{t}$ denotes the act $u \circ\left(\left(w-\mathrm{CE}_{w}\left(l^{\nu}\right)\right) \oplus t f+(1-t) l^{\nu}\right) . \bar{\pi}_{f^{t}, w}^{\nu}=$ $\left\|u \circ\left(\pi_{f^{t}, w}^{\nu} \oplus f_{1}^{t}\right)-\bar{f}_{1}^{t}\right\|$ is the adjusted ambiguity premium $\pi_{f^{t}, w}^{\nu}$.

Figure 2: The Ambiguity Premium

$\tilde{W}$ is differentiable at $\bar{w}$ with a gradient $\frac{1}{\bar{w}} \nu$. Then

$$
\lim _{t \searrow 0} \frac{\tilde{W}(\bar{w})-\tilde{W}\left(\bar{f}_{1}^{t}\right)}{\left\|\bar{f}_{1}^{t}-\bar{w}\right\|}=-\lim _{t \searrow 0} \frac{\tilde{W}\left(\bar{f}_{1}^{t}\right)-\tilde{W}(\bar{w})}{\left\|\bar{f}_{1}^{t}-\bar{w}\right\|}=-\lim _{t \searrow 0} \frac{\left\langle\frac{1}{\bar{w}} \nu, \bar{f}_{1}^{t}-\bar{w}\right\rangle}{\left\|\bar{f}_{1}^{t}-\bar{w}\right\|}
$$

As $\bar{f}_{1}^{t}=t \bar{f}_{1}^{t=1}+(1-t) \bar{w}$ as proven in the beginning of this proof,

$$
-\lim _{t \searrow 0} \frac{\left\langle\frac{1}{\bar{w}} \nu, \bar{f}_{1}^{t}-\bar{w}\right\rangle}{\left\|\bar{f}_{1}^{t}-\bar{w}\right\|}=-\lim _{t \searrow 0} \frac{\left\langle\frac{1}{\bar{w}} \nu, t\left(\bar{f}_{1}^{t=1}-\bar{w}\right)\right\rangle}{t|| \bar{f}_{1}^{t=1}-\bar{w} \|}=\frac{-\lim _{t \searrow 0}\left\langle\nu, \bar{f}_{1}^{t=1}-\bar{w}\right\rangle}{\bar{w}\left\|\bar{f}_{1}^{t=1}-\bar{w}\right\|}=\frac{-\left\langle\nu, \bar{f}_{1}^{t=1}-\bar{w}\right\rangle}{\bar{w}\left\|\bar{f}_{1}^{t=1}-\bar{w}\right\|} .
$$

Yet

$$
\begin{aligned}
& \left\langle\nu, \bar{f}_{1}^{t=1}-\bar{w}\right\rangle=\left\langle\nu, \bar{f}_{1}^{t=1}\right\rangle-\bar{w}= \\
& =-\bar{w}+\sum_{\omega \in \Omega} \sum_{\{x \in \mathbb{R} \mid f(x \mid \omega)>0\}} u\left(w-\mathrm{CE}_{w}\left(l^{\nu}\right)+x\right) \nu(\omega) f(x \mid \omega)= \\
& =-\bar{w}+\sum_{\left\{x \in \mathbb{R} \mid l^{\nu}(x)>0\right\}} u\left(w-\mathrm{CE}_{w}\left(l^{\nu}\right)+x\right) l^{\nu}(x)=-\bar{w}+\bar{w}=0,
\end{aligned}
$$

because $l^{\nu}(x)=\sum_{\omega \in \Omega} \nu(\omega) f(x \mid \omega)$ by the definition of $l^{\nu}$ in (1). Consequently,

$$
\frac{1}{\left\|\bar{f}_{1}^{t=1}-\bar{w}\right\|} \lim _{t \searrow 0} \frac{\tilde{W}(\bar{w})-\tilde{W}\left(\bar{f}_{1}^{t}\right)}{t}=\lim _{t \searrow 0} \frac{\tilde{W}(\bar{w})-\tilde{W}\left(\bar{f}_{1}^{t}\right)}{\left\|\bar{f}_{1}^{t}-\bar{w}\right\|}=0 .
$$

Yet $\lim _{t \searrow 0}\left(\tilde{W}(\bar{w})-\tilde{W}\left(\bar{f}_{1}^{t}\right)\right) / t=0$ yields $\lim _{t \searrow_{0}} d_{1}(t) / t=0$. Assumption 1 and 2 ensure existence of the inverse utility function $u^{-1}$. Denote the sum of the distances in wealth terms by

$$
d_{1}^{*}(t)=\sum_{\omega \in \Omega}\left(u^{-1}\left(\frac{d_{1}(t)}{\left\|\bar{f}_{1}^{t}\right\|} \bar{f}_{1}^{t}(\omega)+\bar{f}_{1}^{t}(\omega)\right)-u^{-1}\left(\bar{f}_{1}^{t}(\omega)\right)\right)
$$

Recall that Assumption 1 ensures that $U$ is continuous and monotone as shown in CerreiaVioglio et al. (2011, Lemma 57). Hence, $\left(d_{1}(t) /\left\|\bar{f}_{1}^{t}\right\|+1\right) \bar{f}_{1}^{t} \in B(\bar{w})$ by the definition of $d_{1}(t)$ and continuity of $U$. By monotonicity and continuity of $U, u \circ\left(d_{1}^{*}(t) \oplus f_{1}^{t}\right) \in B(\bar{w})$, because the definition of $d_{1}^{*}(t)$ guarantees $\left(u \circ\left(d_{1}^{*}(t) \oplus f_{1}^{t}\right)\right)(\omega) \geq\left(d_{1}(t) /\left\|\bar{f}_{1}^{t}\right\|+1\right) \bar{f}_{1}^{t}(\omega)$ for all $\omega \in \Omega$. The ambiguity premium equals $\pi_{f^{t}, w}^{\nu}=\inf \left\{\beta \in \mathbb{R}_{+} \mid u \circ\left(\beta \oplus f_{1}^{t}\right) \in B(\bar{w})\right\}$. 
Hence, $d_{1}^{*}(t) \geq \pi_{f_{1}^{t}, w}^{\nu} \geq 0$. Assumption 1 and the inverse function theorem guarantee that the inverse utility function $u^{-1}$ is differentiable. Then, by l'Hôpital's rule

$$
\begin{aligned}
\lim _{t \searrow 0} \frac{d_{1}^{*}(t)}{t} & =\lim _{t \searrow 0} \frac{\partial d_{1}^{*}(t)}{\partial t}=\sum_{\omega \in \Omega} \frac{\partial u^{-1}}{\partial x}(\bar{w}) \lim _{t \searrow 0}\left(\frac{d_{1}(t) \frac{\bar{f}_{1}^{t}(\omega)}{\left\|f_{1}^{t}\right\|}}{t}+\frac{\partial \bar{f}_{1}^{t}(\omega)}{\partial t}-\frac{\partial \bar{f}_{1}^{t}(\omega)}{\partial t}\right)= \\
& =\sum_{\omega \in \Omega} \frac{\partial u^{-1}}{\partial x}(\bar{w})\left(\lim _{t \searrow 0} d_{1}(t) \frac{\bar{f}_{1}^{t}(\omega)}{\left\|\bar{f}_{1}^{t}\right\| t}\right)
\end{aligned}
$$

As $\bar{f}_{1}^{t}=t f_{1}^{t=1}+(1-t) \bar{w}$, the last line equals

$$
\frac{\partial u^{-1}}{\partial x}(\bar{w}) \sum_{\omega \in \Omega} \lim _{t \searrow 0}\left(\frac{d_{1}(t) \bar{w}}{\left\|\bar{f}_{1}^{t}\right\| t}+d_{1}(t) \frac{f_{1}^{t=1}(\omega)-\bar{w}}{\left\|\bar{f}_{1}^{t}\right\|}\right)=\frac{\partial u^{-1}}{\partial x}(\bar{w}) \frac{\bar{w}}{\left\|\bar{f}_{1}^{t=0}\right\|} \sum_{\omega \in \Omega} \lim _{t \searrow 0} \frac{d_{1}(t)}{t}=0,
$$

$\lim _{t \searrow 0} d_{1}(t)=0$ yields the penultimate equality and $\lim _{t \searrow_{0}} d_{1}(t) / t=0$ yields the last equality. Consequently, $\lim _{t \searrow 0} d_{1}^{*}(t) / t=0$ and $\lim _{t \searrow 0} \pi_{f^{t}, w}^{\nu} / t=0$. This ensures that $L_{f, w}^{\nu}=0$ for the subjective belief $\nu$ and all acts $f$. Therefore there is second-order ambiguity aversion.

Assumption 1 and Cerreia-Vioglio et al. (2011, Lemma 57) ensure that $U$ is quasiconcave. Hence, upper contour sets are convex. Therefore there is a subjective belief at each wealth by Rockafellar (1970, Corollary 11.5.2 and 11.6.1), because analogously to the previous reasoning it is easy to show that such a separating hyperplane is componentwise nonnegative and normalization is feasible. See also Rigotti et al. (2008, p. 1169). In particular, the negation of the statement 'subjective beliefs at wealth $w$ are a singleton' is 'there are several subjective beliefs at wealth $w$.'

Finally, consider the case of several subjective beliefs at wealth $w$. Pick two different subjective beliefs $\lambda \neq \nu$ at wealth $w$. In particular, there is a state of the world $\omega^{*} \in \Omega$, such that

$$
\nu\left(\omega^{*}\right)>\lambda(\omega) .
$$

Now turn to the ambiguity premium $\pi_{f^{t}, w}^{\nu}=\inf \left\{\beta \in \mathbb{R}_{+} \mid u \circ\left(\beta \oplus f_{1}^{t}\right) \in B(\bar{w})\right\}$ for an act $f \in \mathcal{F}$. The ambiguity premium in utility terms $\bar{\pi}_{f^{t}, w}^{\nu}=\left\|u \circ\left(\pi_{f^{t}, w}^{\nu} \oplus f_{1}^{t}\right)-\bar{f}_{1}^{t}\right\|$ is at least as high as the distance between the act $\bar{f}_{1}^{t}$ and the upper contour set $B(\bar{w})$,

$$
\bar{\pi}_{f^{t}, w}^{\nu} \geq \inf \left\{\left\|\bar{f}_{1}^{t}-\bar{g}\right\| \mid \bar{g} \in B(\bar{w})\right\} .
$$

By definition of the subjective beliefs (2), the upper contour set is contained in the halfspace created by the hyperplane determined by the subjective beliefs. In particular, for any subjective belief $P$

$$
\sum_{\omega \in \Omega}(\bar{f}(\omega)-\bar{w}) P(\omega) \geq 0 \quad \forall \bar{f} \in B(\bar{w})
$$

and

$$
B(\bar{w}) \subseteq\left\{\bar{f} \in \overline{\mathcal{F}} \mid \sum_{\omega \in \Omega}(\bar{f}(\omega)-\bar{w}) P(\omega) \geq 0\right\} .
$$


Therefore, the distance between any act $\bar{f} \in \overline{\mathcal{F}}$ and the upper contour set is at least as high as the distance between $\bar{f}$ and the halfspace. According to Krantz (2015, Proof of Theorem 2.34, p.49) the distance to the halfspace is just the length of the orthogonal projection and equals $\max \left\{0, \frac{-1}{\|P\|} \sum_{\omega \in \Omega}(\bar{f}(\omega)-\bar{w}) P(\omega)\right\}$. Therefore,

$\bar{\pi}_{f^{t}, w}^{\nu} \geq \inf \left\{\left\|\bar{f}_{1}^{t}-\bar{g} \mid\right\| \bar{g} \in B(\bar{w})\right\} \geq \inf \left\{|| \bar{f}_{1}^{t}-\bar{g} \| \mid \bar{g} \in\left\{\bar{f} \in \overline{\mathcal{F}} \mid \sum_{\omega \in \Omega}(\bar{f}(\omega)-\bar{w}) P(\omega) \geq 0\right\}\right\}$

and

$$
\bar{\pi}_{f^{t}, w}^{\nu} \geq \max \left\{0, \frac{-1}{\|P\|} \sum_{\omega \in \Omega}\left(\bar{f}_{1}^{t}(\omega)-\bar{w}\right) P(\omega)\right\}=\max \left\{0, \frac{-1}{\|P\|} \sum_{\omega \in \Omega} t\left(\bar{f}_{1}^{t=1}(\omega)-\bar{w}\right) P(\omega)\right\}
$$

for any subjective beliefs $P$. Now consider the act $f$ that yields 1 with probability 1 in state of the world $\omega^{*}$ and 0 with probability 1 in all other states of the world. Remember that (5) determines $\omega^{*}$. Then the lottery $l^{\nu}$ equals

$l^{\nu}(x)=\left\{\begin{array}{ll}\nu\left(\omega^{*}\right), & \text { if } x=1 \\ 1-\nu\left(\omega^{*}\right), & \text { if } x=0 \\ 0, & \text { otherwise }\end{array} \quad\right.$ and $\quad \bar{f}_{1}^{t=1}(\omega)= \begin{cases}u\left(w-\mathrm{CE}_{w}\left(l^{\nu}\right)+1\right), & \text { if } \omega=\omega^{*} \\ u\left(w-\mathrm{CE}_{w}\left(l^{\nu}\right)\right), & \text { otherwise. }\end{cases}$

Inserting into $-\sum_{\omega \in \Omega}\left(\bar{f}_{1}^{t=1}(\omega)-\bar{w}\right) P(\omega)$ yields

$$
\begin{aligned}
& -\sum_{\omega \in \Omega}\left(\bar{f}_{1}^{t=1}(\omega)-\bar{w}\right) P(\omega)=\bar{w}-\sum_{\omega \in \Omega} \bar{f}_{1}^{t=1}(\omega) P(\omega)= \\
& =\left(\nu\left(\omega^{*}\right) u\left(w-\mathrm{CE}_{w}\left(l^{\nu}\right)+1\right)+\left(1-\nu\left(\omega^{*}\right)\right) u\left(w-\mathrm{CE}_{w}\left(l^{\nu}\right)\right)\right)-\sum_{\omega \in \Omega} \bar{f}_{1}^{t=1}(\omega) P(\omega)= \\
& =\left(\nu\left(\omega^{*}\right)-P\left(\omega^{*}\right)\right) u\left(w-\mathrm{CE}_{w}\left(l^{\nu}\right)+1\right)-\left(\nu\left(\omega^{*}\right)-P\left(\omega^{*}\right)\right) u\left(w-\mathrm{CE}_{w}\left(l^{\nu}\right)\right)= \\
& =\left(\nu\left(\omega^{*}\right)-P\left(\omega^{*}\right)\right)\left(u\left(w-\mathrm{CE}_{w}\left(l^{\nu}\right)+1\right)-u\left(w-\mathrm{CE}_{w}\left(l^{\nu}\right)\right)\right)>0
\end{aligned}
$$

The first bracket is positive by (5) for $P=\lambda$. The second bracket is also positive by Assumption 2. Therefore $-\sum_{\omega \in \Omega}\left(\bar{f}_{1}^{t=1}(\omega)-\bar{w}\right) \lambda(\omega)>0$ and

$$
\begin{aligned}
\lim _{t \searrow 0} \bar{\pi}_{f^{t}, w}^{\nu} / t & \geq \lim _{t \searrow 0} \frac{1}{t} \max \left\{0, \frac{-1}{\|\lambda\|} \sum_{\omega \in \Omega} t\left(\bar{f}_{1}^{t=1}(\omega)-\bar{w}\right) \lambda(\omega)\right\}= \\
& =\lim _{t \searrow 0} \frac{1}{t} \frac{-1}{\|\lambda\|} \sum_{\omega \in \Omega} t\left(\bar{f}_{1}^{t=1}(\omega)-\bar{w}\right) \lambda(\omega)=\frac{-1}{\|\lambda\|} \sum_{\omega \in \Omega}\left(\bar{f}_{1}^{t=1}(\omega)-\bar{w}\right) \lambda(\omega)>0
\end{aligned}
$$

for the act $f$. Assumption 1 ensures that $u^{\prime}$ is continuous. Hence, $u^{\prime}$ is bounded on any compact set. In particular, there is a $B \in \mathbb{R}$ with $u^{\prime}(x) \leq B$ for all $x$ in the closure of the convex hull of the union of $w$ and the support of the simple lotteries $f_{1}^{t=1}(\omega),\left(f_{1}^{t=1}+\pi_{f^{t}, w}^{\nu}\right)(\omega)$, and $\left(f_{1}^{t=0}+\pi_{f^{t}, w}^{\nu}\right)(\omega)$ for all $\omega \in \Omega$ and $t \in[0,1]$. Therefore $\pi_{f^{t}, w}^{\nu} \geq \bar{\pi}_{f^{t}, w}^{\nu} /(B\|\mathbf{1}\|)$. Consequently, $L_{f, w}^{\nu}>0$ for an act $f$ and there is first-order ambiguity aversion at $w$. 
Proof of Theorem 2: Consider a given wealth $w \in \mathbb{R}$. Set $\bar{w}=u(w)$. The upper contour set of $U$ at $\bar{f}_{0} \in \overline{\mathcal{F}}$ is denoted $B\left(\bar{f}_{0}\right)=\left\{\bar{f} \in \overline{\mathcal{F}} \mid U(\bar{f}) \geq U\left(\bar{f}_{0}\right)\right\}$. Without loss of generality suppose $0 \notin B(\bar{w})$. Notice that $\overline{\mathcal{F}} \subseteq \mathbb{R}^{\Omega}$ is homeomorphic to a corresponding subspace of $\mathbb{R}^{|\Omega|}$. Therefore I can identify acts $f \in \overline{\mathcal{F}}$ with vectors in $\mathbb{R}^{|\Omega|}$ and vice versa.

Assume second-order ambiguity aversion at $w$. By Theorem 1, there is a unique subjective belief $\nu$ at wealth $w$. The proof of Theorem 1 shows that the function $\tilde{W}$ defined in (3) is differentiable at $\bar{w}$ with a gradient $\frac{1}{\bar{w}} \nu \neq \mathbf{0}$. Obviously, the indifference surface is contained in the upper contour set, i.e.,

$$
\{\bar{f} \in \overline{\mathcal{F}} \mid U(\bar{f})=U(\bar{w})\} \subseteq\{\bar{f} \in \overline{\mathcal{F}} \mid U(\bar{f}) \geq U(\bar{w})\} .
$$

Therefore $\tilde{W}(\bar{f}) \geq-1$ for all $\bar{f} \in \overline{\mathcal{F}}$ with $U(\bar{f})=U(\bar{w})$. Cerreia-Vioglio et al. (2011, Lemma 57) show that the representing utility function $U$ is monotone and continuous under Assumption 1. Assumption 2 ensures strict monotonicity of $U$. Therefore the upper contour sets are closed and $\tilde{W}(\bar{f})=-1$ for all $\bar{f} \in \overline{\mathcal{F}}$ with $U(\bar{f})=U(\bar{w})$. Now suppose to the contrary that there is a $\bar{f} \in \overline{\mathcal{F}}$ with $U(\bar{f}) \neq U(\bar{w})$ and $\tilde{W}(\bar{f})=-1$. Then, by definition of $\tilde{W}, 1 \bar{f}=\bar{f}$ is contained in the closure of $B(\bar{w})$. As the upper contour sets are closed, the closure of $B(\bar{w})$ equals $B(\bar{w})$. Therefore $\bar{f} \in B(\bar{w})$. Yet, $\bar{f} \in B(\bar{w})$ and $U(\bar{f}) \neq U(\bar{w})$ implies $U(\bar{f})>U(\bar{w})$. By continuity of $U$, there is a neighborhood $N$ of $\bar{f}$ such that $U\left(\bar{f}^{\prime}\right)>U(\bar{w})$ for all $\bar{f}^{\prime} \in N$. In particular, there is a $\alpha<1$ such that $\alpha \bar{f} \in N$ contradicting $\tilde{W}(\bar{f})=-1$.

In summary, the indifference surface is the level set of $\tilde{W}$ at the value $-1=\tilde{W}(\bar{w})$. Thus, the indifference surface is differentiable at $\bar{w}$.

Now assume first-order ambiguity aversion at $w$. By Theorem 1, there are several subjective beliefs at $w$. Pick two different subjective beliefs $\nu \neq \lambda$ at $w$. Assume to the contrary that the indifference surface is differentiable. Hence, there is a function $\eta$, so that the indifference surface is the level set of $\eta$ at the value $\eta(\bar{w})$ and $\eta$ is differentiable at $\bar{w}$ with a gradient $\nabla_{\eta}^{\bar{w}} \neq \mathbf{0}$. Suppose without loss of generality that $\left\langle\nabla_{\eta}^{\bar{w}},(1,1, \ldots, 1)\right\rangle \geq 0$.

By definition of the subjective beliefs, $\bar{f} \notin B(\bar{w})$ for all $\bar{f} \in \overline{\mathcal{F}}$ with $\langle\bar{f}, \nu\rangle<\bar{w}$. In particular, the intersection between the indifference surface and the set of all $\bar{f} \in \overline{\mathcal{F}}$ with $\langle\bar{f}, \nu\rangle\left\langle\bar{w}\right.$ must be empty. Assume to the contrary there is no $\beta \in \mathbb{R}$ with $\nu=\beta \nabla_{\eta}^{\bar{w}}$. Then there is a $\bar{g} \in \overline{\mathcal{F}}$ such that $\langle\bar{g}, \nu\rangle\left\langle\bar{w}\right.$ and $\left\langle\bar{g}, \nabla_{\eta}^{\bar{w}}\right\rangle>0$. By definition of the gradient, $\lim _{t \searrow 0} \frac{\eta(\bar{w}+t \bar{f})-\eta(\bar{w})-t\left\langle\bar{f}, \nabla_{\eta}^{\bar{w}}\right\rangle}{t}=0$ for any $\bar{f} \in \overline{\mathcal{F}}$. Hence, there is a $t^{\prime}>0$ with $\eta(\bar{w}+t \bar{g})>\eta(\bar{w})$ for all $t \in\left(0, t^{\prime}\right)$. Hence, the intersection between the indifference surface and the set of all $\bar{f} \in \overline{\mathcal{F}}$ with $\langle\bar{f}, \nu\rangle<\bar{w}$ cannot be empty. Therefore $\nu$ and $\nabla_{\eta}^{\bar{w}}$ are parallel, i.e., there is $\beta \in \mathbb{R}$ with $\nu=\beta \nabla_{\eta}^{\bar{w}}$. Analogously, we can prove that $\lambda$ and $\nabla_{\eta}^{\bar{w}}$ are parallel, i.e., there is $\beta \in \mathbb{R}$ with $\lambda=\beta \nabla_{\eta}^{\bar{w}}$. This result contradicts the fact that $\nu \neq \lambda$ and the fact that probability measures are normalized. Therefore there is no such function $\eta$ and the indifference surface is kinked at $\bar{w}$.

Proof of Lemma 2: Denote the closure of the convex hull of $\Pi$ by $\bar{\Pi}$. This repre- 
sentation is a special case of Uncertainty Averse Preferences with $G(w, P)=w+c(P)$ with $c(P)=0$ if $P \in \bar{\Pi}$ and $c(P)=\infty$ if $P \notin \bar{\Pi}$. By Lemma 4, the subjective beliefs are $\arg \inf _{P \in \Delta \Omega} G(w, P)=\bar{\Pi}$ for every $w \in \mathbb{R}$. Hence, there are several subjective beliefs if $\Pi$ is not a singleton. By Theorem 1, MEU preferences exhibit first-order ambiguity aversion.

Proof of Lemma 3: Rigotti et al. (2008, Proposition 5) show that there is a unique subjective belief at each wealth, namely $\mathbb{E}_{\mu} P$. By Theorem 1 , the preferences exhibit second-order ambiguity aversion. Maccheroni et al. (2011) discuss the orders of risk and ambiguity aversion in Smooth Ambiguity-Averse Preferences in detail. Cerreia-Vioglio et al. (2015) characterize subjective beliefs for any locally Lipschitz preferences.

Proof of Lemma 4: Consider a wealth level $w$. Cerreia-Vioglio et al. (2011, Proposition 11(i)) show that the subjective beliefs at wealth $w$ equal $\arg \inf _{P \in \Delta \Omega} G(\bar{w}, P)$. Notice that the proof of Cerreia-Vioglio et al. (2011, Proposition 11(i)) does not require their axiom A.6. Therefore my framework satisfies all the required conditions. Theorem 1 yields the statement of the proposition.

Consider an endowment of $w \in \mathbb{R}$ and an act $f \in \mathcal{F}$. An individual chooses the optimal $t \geq 0$ for the act $w \oplus(t * f)$.

Theorem 5. Suppose Assumption 1-2 hold. If the individual chooses $t>0$, there is a $t^{\prime}>0$, such that $\mathbb{E}_{\nu}\left(u \circ\left(w \oplus\left(t^{\prime} * f\right)\right)\right) \geq u(w)$ for any subjective belief $\nu$ at $w$. If there is second-order ambiguity aversion at $w$ and the subjective beliefs $\nu$ at $w$ yields a positive expectation of the act, $\mathbb{E}_{\nu}(i d \circ f)>0$, the individual chooses $t>0$.

Proof of Theorem 5: Consider an individual who prefers $w \oplus\left(t^{\prime} * f\right)$ to $w$ with $t^{\prime}>0$. Then $u \circ\left(w \oplus\left(t^{\prime} * f\right)\right) \in B(u(w))$. The proof of Theorem 1 shows that there is a subjective belief at wealth $w$. By definition of the subjective belief $\nu$ at $w$,

$$
\sum_{\omega \in \Omega} \nu(\omega)\left(u \circ\left(w \oplus\left(t^{\prime} * f\right)\right)\right)(\omega) \geq \sum_{\omega \in \Omega} \nu(\omega) u(w)=u(w)
$$

Therefore $\mathbb{E}_{\nu}\left(u \circ\left(w \oplus\left(t^{\prime} * f\right)\right)\right) \geq u(w)$. This proves the first part of the theorem.

For the second part of the theorem, assume second-order ambiguity aversion at $w$ and that there is a subjective belief $\nu$ at $w$ that yields a positive expectation of the act, $\mathbb{E}_{\nu}(i d \circ f)=\sum_{\omega \in \Omega}\left(\nu(\omega)\left(\sum_{x: f(x \mid \omega)>0} x f(x \mid \omega)\right)\right)>0$. Without loss of generality, suppose $u(w)>0$. The proof of Theorem 1 shows that the function $\tilde{W}$ is differentiable at $u(w)$ with a gradient $\frac{1}{u(w)} \nu$. By the definition of the gradient, $\tilde{W}\left(u \circ\left(w \oplus\left(t^{\prime} * f\right)\right)\right)$ is increasing in $t$ for small $t$ if

$$
\left\langle\frac{1}{u(w)} \nu,\left.\frac{\partial u \circ(w \oplus(t * f))}{\partial t}\right|_{t=0}\right\rangle>0
$$


By Assumption $1 u$ is differentiable and

$$
\begin{aligned}
& \left.\frac{\partial \sum_{x: f(x \mid \omega)>0} u(w+t x) f(x \mid \omega)}{\partial t}\right|_{t=0}=\left.\sum_{x: f(x \mid \omega)>0} u^{\prime}(w+t x) x f(x \mid \omega)\right|_{t=0}= \\
& =u^{\prime}(w) \sum_{x: f(x \mid \omega)>0} x f(x \mid \omega)=u^{\prime}(w)(i d \circ f)(\omega)
\end{aligned}
$$

for all $\omega \in \Omega$. Therefore

$$
\left\langle\frac{1}{u(w)} \nu,\left.\frac{\partial u \circ(w \oplus(t * f))}{\partial t}\right|_{t=0}\right\rangle=\frac{1}{u(w)}\left\langle\nu,\left.\frac{\partial u \circ(w \oplus(t * f))}{\partial t}\right|_{t=0}\right\rangle=\frac{u^{\prime}(w)}{u(w)} \mathbb{E}_{\nu}(i d \circ f)>0
$$

by assumption. Hence, there is a $t^{\prime}>0$ with $\tilde{W}\left(u \circ\left(w \oplus\left(t^{\prime} * f\right)\right)\right)>\tilde{W}(u(w))$. Assumption 2 ensures that the individual then prefers $t^{\prime}$ to her endowment. This concludes the second part of the proof.

Proof of Theorem 3: Suppose the individual chooses $t>0$. Theorem 5 above shows that $\mathbb{E}_{\nu}(u \circ(w \oplus(t * f))) \geq u(w)$ for any subjective belief $\nu$ at wealth $w$. Assumption 3 ensures that $u$ is concave. Jensen's inequality ensures

$$
u\left(\mathbb{E}_{\nu}(i d \circ(w \oplus(t * f)))\right) \geq \mathbb{E}_{\nu}(u \circ(w \oplus(t * f))) \geq u(w) .
$$

If, on the one hand, at least one of the inequalities in (6) is a strict inequality, we have $u\left(\mathbb{E}_{\nu}(i d \circ(w \oplus(t * f)))\right)>u(w)$. Then, as $u$ is increasing under Assumption 2, (6) implies $E_{\nu}(i d \circ(w \oplus(t * f)))>w$ and, hence, $E_{\nu}(i d \circ f)>0$.

If, on the other hand, $u\left(\mathbb{E}_{\nu}(i d \circ(w \oplus(t * f)))\right)=u(w), \mathbb{E}_{\nu}(i d \circ(w \oplus(t * f)))=w$ because $u$ is strictly increasing under Assumption 2. In particular, $\mathbb{E}_{\nu}(i d \circ f)=0$. As $i d \circ f \neq \mathbf{0}$ by assumption, there are states $\omega_{1}, \omega_{2} \in \Omega$ with $(i d \circ f)\left(\omega_{1}\right)<0$ and $(i d \circ f)\left(\omega_{2}\right)>0$. Then the strict concavity of $u$ by Assumption 3 and Jensen's inequality yield $u\left(\mathbb{E}_{\nu}(i d \circ(w \oplus(t * f)))\right)>\mathbb{E}_{\nu}(u \circ(w \oplus(t * f)))$ contradicting $u\left(\mathbb{E}_{\nu}(i d \circ(w \oplus(t * f)))\right)=$ $u(w)$. This proves that the condition is necessary.

Theorem 5 above proves the sufficiency part for second-order ambiguity aversion. For the sufficiency part for first-order ambiguity aversion assume to the contrary that any subjective beliefs $\nu$ at wealth $w$ yield a positive expectation of the act, $\mathbb{E}_{\nu}(i d \circ f)>0$, but $t=0$ is optimal. Then the individual (weakly) prefers $w$ to any $w \oplus(t * f)$ with $t>0$. By Assumptions 1 and 2 , there is a $P \in \Delta \Omega$, such that $\mathbb{E}_{P}(i d \circ f)=0$ and $U(u \circ g)<U(u \circ w)$ for all $g \in \mathcal{F}$ with $\mathbb{E}_{P}(i d \circ g)<w$. This implies that $\mathbb{E}_{P}(i d \circ g) \geq w$ for all $g \in \mathcal{F}$ with $U(u \circ g) \geq U(u \circ w)$. Therefore by definition $P$ is a subjective belief at wealth $w$ with $\mathbb{E}_{P}(i d \circ f)=0$. This yields a contradiction.

Proof of Theorem 4: Finally, consider an individual who is first-order ambiguity averse at wealth $w$ and risk averse with a concave $u$. Then by Theorem 1 there are at least two subjective beliefs at $w$. Denote them by $\lambda$ and $\nu$. In particular, there is a state of the world $\omega^{*}$ with $\lambda\left(\omega^{*}\right)<\nu\left(\omega^{*}\right)$. Notice that $\frac{1-\nu\left(\omega^{*}\right)}{\nu\left(\omega^{*}\right)}<\frac{1-\lambda\left(\omega^{*}\right)}{\lambda\left(\omega^{*}\right)}$. Pick a $x \in \mathbb{R}$ with 
$\frac{1-\nu\left(\omega^{*}\right)}{\nu\left(\omega^{*}\right)}<x<\frac{1-\lambda\left(\omega^{*}\right)}{\lambda\left(\omega^{*}\right)}$. Now consider the act $g \in \mathcal{F}$ that pays $x$ with probability 1 in the state $\omega^{*}$ and otherwise pays -1 with probability 1 . The act $g$ has a positive expectation under the subjective belief $\nu$

$$
\mathbb{E}_{\nu}(i d \circ g)=\nu\left(\omega^{*}\right) x-\left(1-\nu\left(\omega^{*}\right)\right)>0,
$$

but a negative expectation under the subjective belief $\lambda$

$$
\mathbb{E}_{\lambda}(i d \circ g)=\lambda\left(\omega^{*}\right) x-\left(1-\lambda\left(\omega^{*}\right)\right)<0 .
$$

Jensen's inequality ensures

$$
\mathbb{E}_{\lambda}(u \circ(w \oplus(t * g))) \leq u\left(\mathbb{E}_{\lambda}(i d \circ(w \oplus(t * g)))\right)=u\left(w+t \mathbb{E}_{\lambda}(i d \circ g)\right)<u(w)
$$

for $t>0$ because $\mathbb{E}_{\lambda}(i d \circ g)<0, u$ is concave by assumption and strictly increasing under Assumption 2. By the definition of the subjective belief $\lambda$, therefore $u \circ(w \oplus(t * g)) \notin$ $B(u(w))$ for all $t>0$ and the individual chooses $t=0$. By continuity of the expectation, there is a open neighborhood $N$ of $g$, such that $\left.\mathbb{E}_{\nu}\left(i d \circ g^{\prime}\right)\right)>0$ and $\left.\mathbb{E}_{\lambda}\left(i d \circ g^{\prime}\right)\right)<0$ for all acts $g^{\prime} \in N$. Hence, $u \circ\left(w \oplus\left(t * g^{\prime}\right)\right) \notin B(u(w))$ for all $t>0$ and $g^{\prime} \in N$. As the set $N$ is nonempty and open, $N$ has positive mass for any atomless measure with full support on $\mathcal{F}$.

Proof of Lemma 5: Suppose $\nu$ is a subjective belief at wealth $r w$. The expected return under the subjective belief $\nu$ of the asset $f$ at price $p$ equals $\mathbb{E}_{\nu}(i d \circ f) / p$ and is either higher, equal or lower than the the return $r$ of the risk-free asset. According to Corollary 1 , the individual either buys or sells a fraction of the asset $f$ at price $p$ with second-order ambiguity aversion at $r w$ whenever $\mathbb{E}_{\nu}(i d \circ f) \neq r p$ and $i d \circ f$ is not constant. The set of assets $f$ with $\mathbb{E}_{\nu}(i d \circ f)=r p$ or constant $i d \circ(-r p \oplus f)$ is a null set in the asset space $\mathcal{F}$. Hence, the individual generically buys or sells a positive amount of the asset $f$. With first-order ambiguity aversion at $r w$, Theorem 4 and Corollary 1 guarantee that there is a set of assets with positive mass so that the individual avoids any investment in these assets.

Proof of Corollary 1: Suppose $\nu$ is a subjective belief at wealth $r w$. The expected return under the subjective belief $\nu$ of the asset $f$ at price $p$ equals $\mathbb{E}_{\nu}(i d \circ f) / p$. Consider the act $g=(-r p) \oplus f$. Then $i d \circ g$ is not constant, as $i d \circ f$ is not constant. Applying Theorem 3 to the act $g$ at wealth $r w$ shows that the individual with second-order ambiguity aversion at $r w$ buys the asset if and only if $\mathbb{E}_{\nu}(i d \circ f)>r p$. Theorem 4 at wealth $r w$ yields the statement of the corollary for first-order ambiguity aversion at $r w$.

Proof of Lemma 6: Suppose $\nu$ is a subjective belief at wealth $w-p$. According to Corollary 2, the individual with second-order ambiguity aversion at $w-p$ buys full insurance coverage, $\alpha=1$ if and only if $\left|\mathbb{E}_{\nu}(i d \circ L)\right|=p$. With first-order ambiguity aversion at $w-p$, Theorem 4 and Corollary 2 guarantee that there are losses such that 
full insurance, $\alpha=1$, is optimal at $\left|\mathbb{E}_{\nu}(i d \circ L)\right| \neq p$.

Proof of Corollary 2: Suppose $\nu$ is a subjective belief at wealth $w-p$. Consider the act $g=p \oplus L$. Then $i d \circ g$ is not constant, as $i d \circ L$ is not constant. Applying Theorem 3 to the act $g$ at wealth $w-p$ with second-order ambiguity aversion at $w-p$ shows that $\alpha<1$ if and only if $\left|\mathbb{E}_{\nu}(i d \circ L)\right|<p$. In addition, it shows that $\alpha>1$ if and only if $\left|\mathbb{E}_{\nu}(i d \circ L)\right|>p$. Therefore full insurance, $\alpha=1$, is only feasible for $\left|\mathbb{E}_{\nu}(i d \circ L)\right|=p$. Notice that full insurance, $\alpha=1$, here corresponds to $t=0$ in the theorem. Theorem 4 at wealth $w-p$ shows that full insurance, i.e., $\alpha=1$, is optimal for some losses $L$ with $\left|\mathbb{E}_{\nu}(i d \circ L)\right| \neq p$ and first-order ambiguity aversion at $w-p$.

Proof of Corollary 3: Suppose $\nu$ is a subjective belief at wealth $w-c(1)$. By convexity of $c, c(e) \geq c(1)+(1-e) c^{\prime}(1)$ for all efforts $e \in[0,1]$. Consider the act $g=c^{\prime}(1) \oplus L$. Then $i d \circ g$ is not constant, as $i d \circ L$ is not constant. Applying Theorem 3 to the act $g$ at wealth $w-c(1)$ for second-order ambiguity aversion at $w-c(1)$ shows that $t>0$ if and only if $c^{\prime}(1)>\left|\mathbb{E}_{\nu}(i d \circ L)\right|$. Assumption 1 ensures monotonicity of preferences. By monotonicity of preferences and convexity of $c, e<1$ only if $t>0$. Suppose to the contrary, that for the linear act $t>0$ is optimal and for the convex act $e=1$ is optimal. Then there is an $\epsilon>0$ such that $c^{\prime}(1-\epsilon)>\left|\mathbb{E}_{\nu}(i d \circ L)\right|$ and, hence, also $t>0$ is optimal when facing the new act $g^{\prime}=c^{\prime}(1-\epsilon) \oplus L$. Yet, $c(e) \leq c(1)+(1-e) c^{\prime}(1-\epsilon)$ for $e$ in a neighborhood of 1 . Monotonicity of preferences yields an optimal $e<1$ contradicting the initial assumption. Consequently, $e<1$ if and only if $t>0$. Theorem 4 at wealth $w-c(1)$ shows that perfect prevention, i.e., $e=1$, is optimal for some losses $L$ with $c^{\prime}(1)>\left|\mathbb{E}_{\nu}(i d \circ L)\right|$ and first-order ambiguity aversion at $w-c(1)$.

Proof of Corollary 4: Consider second-order ambiguity aversion at wealth $\mathbb{E}_{\mu^{0}} W(y)$ with subjective beliefs $\nu$ at wealth $\mathbb{E}_{\mu^{0}} W(y)$. Analogously to the proof of Theorem 5 , differentiability of $\tilde{W}$ ensures that $\tilde{W}\left(u \circ\left(-c(e) \oplus w\left(y^{e}\right)\right)\right)$ is increasing in $e$ for small $e$ if there is an $\epsilon^{\prime}>0$ such that

$$
\sum_{\omega \in \Omega} \nu(\omega)\left(\left(u \circ\left(-c(e) \oplus w\left(y^{e}\right)\right)\right)(\omega)-\left(u \circ w\left(y^{0}\right)\right)(\omega)\right)>0
$$

for all $e \in\left(0, \epsilon^{\prime}\right)$. Consistency guarantees that $\mu^{e}$ equals the distribution of $y^{e}$ under $\nu$. Therefore the previous condition is equivalent to existence of an $\epsilon^{\prime}>0$ such that

$$
\sum_{y \in Y} \mu^{e}(y) u(W(y)-c(e))-\left(u \circ w\left(y^{0}\right)\right)(\omega)>0
$$

for all $e \in\left(0, \epsilon^{\prime}\right)$. This condition is exactly for a subjective expected utility maximizer with risk index $u$ and beliefs $\mu^{e}$ to prefer $-c(e) \oplus w\left(y^{e}\right)$ to $w\left(y^{0}\right)$ for all $e \in\left[0, \epsilon^{\prime}\right]$. Therefore $\tilde{W}$ is increasing in this direction. The condition on $\left\|u \circ\left(-c(e) \oplus w\left(y^{e}\right)\right)-u \circ w\left(y^{0}\right)\right\|$ ensures that we are getting close enough to $u \circ w\left(y^{0}\right) \in \overline{\mathcal{F}}$ for small $e$. Consequently, there is an $\epsilon^{\prime}>0$ such that $\tilde{W}\left(u \circ\left(-c(e) \oplus w\left(y^{e}\right)\right)\right)>\tilde{W}\left(u \circ w\left(y^{0}\right)\right)$ for all $e \in\left(0, \epsilon^{\prime}\right)$. The agent with 
second-order ambiguity aversion prefers to exert positive effort.

With first-order ambiguity aversion, however, it may be optimal for the agent to choose zero effort. Theorem 1 shows that there are several subjective beliefs. In particular, there might be a subjective belief $\lambda$ and an $\epsilon^{\prime}>0$ with

$$
\sum_{\omega \in \Omega} \lambda(\omega)\left(\left(u \circ\left(-c(e) \oplus w\left(y^{e}\right)\right)\right)(\omega)-\left(u \circ w\left(y^{0}\right)\right)(\omega)\right)<0
$$

for all $e \in\left(0, \epsilon^{\prime}\right)$. The definition of the subjective beliefs ensures that the agent chooses $e=0$.

\section{A.2 Orders of Risk Aversion}

Consider a classical expected utility representation with a twice differentiable von-Neumann-Morgenstern utility index and a lottery $l$. Then the risk premium is proportional to the variance for small risks, as already recognized by Pratt (1964, p. 126). Consequently, the risk premium only contains terms of second order. This yields risk-neutral behavior for small stakes and is called second-order risk aversion. Segal and Spivak (1990) introduce the notion of first-order risk aversion if the risk premium is proportional to the standard deviation. They consider a lottery $l$ with $\mathbb{E}(l)=0$. Assume a risk-averse individual chooses between a zero payoff and the lottery $l$. Given her risk aversion, the individual prefers the certain payoff to the lottery, $0 \succcurlyeq l$. If preferences are continuous in wealth, there exists a payment $\hat{\pi}_{l, 0}$, such that the individual is indifferent between 0 and the lottery $\hat{\pi}_{l, 0} \oplus l$ :

$$
0 \sim \hat{\pi}_{l, 0} \oplus l
$$

$\hat{\pi}_{l, 0}$ is called the risk premium of the lottery $l$. There are different definitions of the risk premium. Sometimes it is the transfer that is subtracted from the endowment in order to make the individual indifferent to the lottery. See also Schlesinger's (1997) footnote 2 for a discussion of the different definitions. To capture small risks, consider $t \geq 0$ and $\hat{\pi}_{t * l, 0}$, such that $0 \sim \hat{\pi}_{t * l, 0} \oplus(t * l)$. Thus, the payment $\hat{\pi}_{t * l, 0}$ makes the individual indifferent between the lottery $t * l$ and the sure zero payoff. If the risk disappears, the risk premium vanishes and $\hat{\pi}_{t * l, 0} \rightarrow 0$ for $t \searrow 0$. According to Segal and Spivak (1990), the individual exhibits first-order risk aversion if

$$
\lim _{t \searrow 0} \frac{1}{t} \hat{\pi}_{t * l, 0}>0
$$

On the other hand, she is second-order risk averse if the risk premium is proportional to the variance and declines quadratically. Then

$$
\lim _{t \searrow 0} \frac{1}{t} \hat{\pi}_{t * l, 0}=0 \quad \text { and } \quad \lim _{t \searrow 0} \frac{1}{t^{2}} \hat{\pi}_{t * l, 0}>0 .
$$

It is common to subsume all higher risk orders under second-order risk aversion and neglect the second condition. Consequently, an individual is second-order risk averse if and only if she behaves in a risk-neutral way with regard to infinitesimal risks. Segal 
and Spivak (1990) prove that expected utility theory with a differentiable utility index implies second-order risk aversion. If the utility index is nondifferentiable at wealth $w$, expected utility theory features first-order risk aversion. Risk aversion implies a concave utility index $u$. Hence, there are right and left derivatives of $u$ at $w$ and

$$
\left.\frac{\partial \hat{\pi}_{t * l, 0}}{\partial t}\right|_{t=0^{+}}=\left(1-\frac{u_{+}^{\prime}(w)}{u_{-}^{\prime}(w)}\right) \int_{\{x \in \mathbb{R} \mid x>0\}} x \mathrm{~d} l(x) .
$$

The same holds in the setting of Machina (1982), in which local utility functions allow approximating non-standard behavior in an expected-utility framework. Segal and Spivak (1997) show that also in this case non-differentiability of the local utility functions is equivalent to first-order risk aversion. See also Nielsen (1999) for a detailed discussion of the effect of a differentiable risk index $u$ on the order of risk aversion.

\section{References}

Ahn, D., Choi, S., Gale, D., and Kariv, S. (2014). Estimating ambiguity aversion in a portfolio choice experiment. Quantitative Economics, 5(2):195-223.

Anscombe, F. J. and Aumann, R. J. (1963). A Definition of Subjective Probability. Annals of Mathematical Statistics, 34(1):199-205.

Cerreia-Vioglio, S., Maccheroni, F., Marinacci, M., and Montrucchio, L. (2011). Uncertainty averse preferences. Journal of Economic Theory, 146(4):1275-1330.

Cerreia-Vioglio, S., Maccheroni, F., Marinacci, M., and Montrucchio, L. (2012). Probabilistic sophistication, second order stochastic dominance and uncertainty aversion. Journal of Mathematical Economics, 48:271-283.

Cerreia-Vioglio, S., Maccheroni, F., Marinacci, M., and Rustichini, A. (2015). The structure of variational preferences. Journal of Mathematical Economics, 57:12-19.

Chateauneuf, A. and Faro, J. H. (2009). Ambiguity through confidence functions. Journal of Mathematical Economics, 45(9-10):535-558.

Chew, S. H. and Sagi, J. S. (2008). Small worlds: Modeling attitudes toward sources of uncertainty. Journal of Economic Theory, 139(1):1-24.

de Finetti, B. (1937). La prévision: ses lois logiques, ses sources subjectives. Annales de l'Institut Henri Poincaré, 7:1-68. Translation reprinted in H.E. Kyburg and H.E. Smokler (eds.) (1980), Studies in Subjective Probability, New York.

Dow, J. and Werlang, S. R. d. C. (1992). Uncertainty Aversion, Risk Aversion, and the Optimal Choice of Portfolio. Econometrica, 60(1):197-204.

Epstein, L. G. (2010). A paradox for the 'smooth ambiguity' model of preference. Econometrica, $78(6): 2085-2099$.

Epstein, L. G. and Schneider, M. (2010). Ambiguity and Asset Markets. Annual Review of Financial Economics, 2(1):315-346.

Ergin, H. and Gul, F. (2009). A theory of subjective compound lotteries. Journal of Economic Theory, 144(3):899-929.

Ghirardato, P. (1994). Agency theory with non-additive uncertainty. Mimeo, University of California at Berkeley. 
Ghirardato, P., Maccheroni, F., and Marinacci, M. (2004). Differentiating Ambiguity and Ambiguity Attitude. Journal of Economic Theory, 118:133-173.

Ghirardato, P. and Marinacci, M. (2002). Ambiguity Made Precise: A Comparative Foundation. Journal of Economic Theory, 102(2):251-289.

Gilboa, I. and Schmeidler, D. (1989). Maxmin Expected Utility with Non-Unique Prior. Journal of Mathematical Economics, 18(2):141-153.

Gollier, C. (2011). Portfolio Choices and Asset Prices: The Comparative Statics of Ambiguity Aversion. Review of Economic Studies, 78(4):1329-1344.

Hansen, L. P. and Sargent, T. J. (2001). Robust control and model uncertainty. American Economic Review, 91(2):60-66.

Holmström, B. (1979). Moral Hazard and Observability. Bell Journal of Economics, 10(1):74-91.

Kellner, C. (2015). Tournaments as a response to ambiguity aversion in incentive contracts. Journal of Economic Theory, 159(A):627-655.

Klibanoff, P., Marinacci, M., and Mukerji, S. (2005). A smooth model of decision making under ambiguity. Econometrica, 73(6):1849-1892.

Krantz, S. (2015). Convex Analysis. CRC Press.

Kudryavtsev, L. D. (1990). Implicit function. In Hazewinkel, M., editor, Encyclopedia of Mathematics. Kluwer Academic Publishers, Dordrecht.

Maccheroni, F., Marinacci, M., and Ruffino, D. (2011). Does Uncertainty Vanish in the Small? The Smooth Ambiguity Case. IGIER Working Papers, 391.

Maccheroni, F., Marinacci, M., and Ruffino, D. (2013). Alpha as Ambiguity: Robust MeanVariance Portfolio Analysis. Econometrica, 81(3):1075-1113.

Maccheroni, F., Marinacci, M., and Rustichini, A. (2006). Ambiguity Aversion, Robustness, and the Variational Representation of Preferences. Econometrica, 74(6):1447-1498.

Machina, M. J. (1982). "Expected Utility" Analysis without the Independence Axiom. Econometrica, 50(2):277-323.

Mandler, M. (2013). Endogenous Indeterminacy and Volatility of Asset Prices Under Ambiguity. Theoretical Economics, 8(3):729-750.

Mossin, J. (1968). Aspects of Rational Insurance Purchasing. Journal of Political Economy, $76(4): 553-568$.

Mukerji, S. (1998). Ambiguity Aversion and the Incompleteness of Contractual Form. American Economic Review, 88(5):1207-1232.

Mukerji, S. and Tallon, J.-M. (2001). Ambiguity Aversion and Incompleteness of Financial Markets. Review of Economic Studies, 68(4):883-904.

Mukerji, S. and Tallon, J.-M. (2004). Ambiguity aversion and the absence of wage indexation. Journal of Monetary Economics, 51(3):653-670.

Nau, R. F. (2006). Uncertainty Aversion with Second-Order Utilities and Probabilities. Management Science, 52(1):136-145.

Nielsen, L. T. (1999). Differentiable von Neumann-Morgenstern utility. Economic Theory, 14:285296.

Pratt, J. W. (1964). Risk Aversion in the Small and in the Large. Econometrica, 32(1/2):122-136. 
Ramsey, F. (1931). Truth and probability. Foundations of Mathematics and other Logical Essays. Reprinted in H.E. Kyburg and H.E. Smokler (eds.) (1980), Studies in Subjective Probability, New York.

Rigotti, L. and Shannon, C. (2012). Sharing Risk and Ambiguity. Journal Economic Theory, 147(5):2028-2039.

Rigotti, L., Shannon, C., and Strzalecki, T. (2008). Subjective Beliefs and Ex-Ante Trade. Econometrica, 76(5):1167-1190.

Rinaldi, F. (2009). Endogenous incompleteness of financial markets: The role of ambiguity and ambiguity aversion. Journal of Mathematical Economics, 45(12):880-901.

Rockafellar, R. T. (1970). Convex Analysis. Princeton University Press, Princeton.

Savage, L. J. (1954). The foundations of statistics. Wiley, New York.

Schlesinger, H. (1997). Insurance Demand without the Expected-Utility Paradigm. Journal of Risk and Insurance, 64(1):19-39.

Schmeidler, D. (1989). Subjective Probability and Expected Utility without Additivity. Econometrica, $57(3): 571-587$.

Segal, U. and Spivak, A. (1990). First Order Versus Second Order Risk Aversion. Journal of Economic Theory, 51(1):111-125.

Segal, U. and Spivak, A. (1997). First-Order Risk Aversion and Non-Differentiability. Economic Theory, 9(1):179-183.

Seo, K. (2009). Ambiguity and Second-Order Belief. Econometrica, 77(5):1575-1605.

Williams, S. R. and Radner, R. (1988). Efficiency in Partnership When The Joint Output is Uncertain. Center for Mathematical Studies in Economics and Management Science Discussion Papers, 760 .

Yaari, M. E. (1969). Some remarks on measures of risk aversion and on their uses. Journal of Economic Theory, 1(3):315-329. 